\title{
Reliability Analysis and Control Strategy Design on Preventive Maintenance of Repairable Equipment
}

\author{
Yongqiang Kang and Hongsheng Su \\ School of Automation and Electrical Engineering, Lanzhou Jiaotong University, \\ Lanzhou 730070, China \\ kangyong137@163.com,shsen@163.com
}

\begin{abstract}
In view of the limitations that the optimal examining items do not have dynamic and real-time performance in the traditional Preventive Maintenance (PM) model of repairable devices, a dynamic and real-time control strategy that selecting checking rate as control variable to control the dynamic behaviour of maintenance system is proposed based on the actual state of devices. The mathematical model of maintenance system is established, some performance indexes of maintenance system including the steady-state availability and the Mean Time To Failure (MTTF) are calculated. And then the control laws on the checking rate are designed. In the end, devices state evaluation is performed and the control errors and the selection of control timing are analyzed and given out. The essence of the strategy proposed in this paper is to achieve the expected steady-state behaviour by controlling the dynamic behavior of maintenance system and reduce the possibility of corrective maintenance by modifying MTTF of maintenance system, which will guarantee reliable completion of target task and reduce maintenance cost. Researches indicate that the strategy proposed in this paper is very effective to improve the utilization of devices and guide the maintenance practice activities of enterprises.
\end{abstract}

Keywords: Reliability, checking rate, control strategy, state evaluation, MTTF

\section{Introduction}

Equipment maintenance system as a dynamic and complex system, how to control the operation process of maintenance system is very important to ensure the normal work of equipment. To improve utilization of equipment and prolong service life of equipment, the maintenance that aims at precise guarantee requires that the operation mode of relevant maintenance system must change from traditional PM strategy to dynamic maintenance strategy based on actual condition of equipment [1].

At present, preventive maintenance strategy has regular maintenance and CBM (Condition Based Maintenance) [2-5]. The shortcoming of regular maintenance is that the optimal examining item or frequency will not change once determined in advance. And regular maintenance isn't dynamic and real-time and can't reflect the actual state of equipment. CBM models have PHM (Proportional Hazards Model), PIM (Proportional Intensities Model), FTM (Failure Threshold Model), DTM (Delay Time Model) and Markov model [6-8], where PIM is the expansion of PHM. PHM considers unrepairable system or complete maintenance condition of repairable system and PIM considers imperfect maintenance condition of repairable system, but difficulty is that they need enough failure data and covariate information to estimate model parameters. FTM needs to specify the failure threshold $c$ of element and replace or repair element when the current state $Y$ of the element matches $Y>c$, but that needs enough observation information and how to choose the failure threshold $c$ is a difficult thing for maintenance crews. DTM 
divides the failure process of element into two stages, the first stage denotes the time from beginning work to beginning failure and the second stage denotes the time from beginning failure to complete failure. We call the second stage delay time and replace or repair element when element is in second stage, but this model requires element has obvious defect stage. Markov model divides the deterioration process of element into $n$ discrete state and makes a decision of replacing or repairing in every state, but this model is complex to be resolved and is difficult to be applied practically.

In view of the above issues, the paper proposes a dynamic and real-time control strategy that selecting checking rate as control variable to control the dynamic behaviour of maintenance system, establishes mathematical model of maintenance system and calculates the steady-state availability and MTTF of the maintenance system. And then the dynamic control laws on the checking rate are designed and devices state evaluation is performed and the control errors and the selection of control timing are analyzed. This maintenance strategy proposed in the paper aims at controlling the dynamic behaviour of the system to achieve the expected steadystate behaviour by selecting the checking rate as control variable and modifying MTTF of the maintenance system as well as reducing cost of corrective maintenance. Compared with the existing traditional PM strategy and CBM strategy, this maintenance strategy proposed in the paper has simple mathematical model, its control laws on the checking rate is dynamic and real-time and it is easy to collect data as well as this strategy can be applied practically, which is very effective to improve the utilization of devices and provide theoretical support for management problems of the large complex equipments.

\section{Model Description}

In order to establish the life cycle model of repairable devices, we do the following assumptions [9-10].

Hypothesis 1. Whether device is in working state or in storage state, the failure of device can be detected out.

Hypothesis 2. The probability of device from state $S_{i}$ at time $t$ to state $S_{j}$ at time $t+\Delta t$ is only proportional to the time interval $\Delta t$ and the transition rate is a constant which does not depend on the time $t$ and $\Delta t$.

Hypothesis 3. Maintenance will not change failure rate of device, but it will prolong life of device.

Based on above assumptions, the life cycle model of repairable devices can be denoted by using the state transition diagram as shown in Fig. 1 and its symbolic meaning is below.

In Figure 1, S1 denotes that device is in storage state in the warehouse, S2 denotes that device is in working state and S3 denotes that device is in checking state or corrective maintenance state. $\mu_{1}$ is the maintenance rate from state $S_{3}$ to state $S_{1}$ and $\mu_{2}$ is the maintenance rate from state $S_{3}$ to state $S_{2}$. $\lambda_{i}$ denotes the state transition rate. Among them, $\lambda_{1}$ denotes the transition rate from state $S_{2}$ to state $S_{1}, \lambda_{2}$ denotes the transition rate from state $S_{1}$ to state $S_{2,} \lambda_{3}$ denotes the transition rate from state $S_{1}$ to state $S_{3}$ and $\lambda_{4}$ denotes the transition rate from state $S_{2}$ to state $S_{3}$. Let $\lambda_{3}=v_{1}+u_{1}$ and $\lambda_{4}=v_{2}+u_{2}$, where $v_{1}$ and $u_{1}$ denote respectively the failure rate and the checking rate of the stored device, $v_{2}$ and $u_{2}$ denote respectively the failure rate and the checking rate of the working device. 


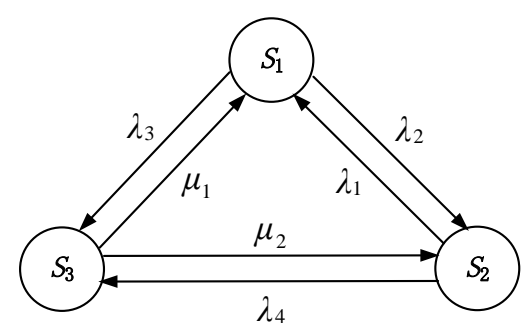

Figure 1. State Transition Diagram of Repairable Devices

\section{Reliability Analysis}

According to Figure 1 and reliability theory [11], we have

$$
\left.\begin{array}{c}
\frac{\mathrm{d} x_{1}(t)}{\mathrm{d} t}=-\left(\lambda_{2}+\lambda_{3}\right) x_{1}(t)+\lambda_{1} x_{2}(t)+\mu_{1} x_{3}(t) \\
\frac{\mathrm{d} x_{2}(t)}{\mathrm{d} t}=\lambda_{2} x_{1}(t)-\left(\lambda_{1}+\lambda_{4}\right) x_{2}(t)+\mu_{2} x_{3}(t) \\
\frac{\mathrm{d} x_{3}(t)}{\mathrm{d} t}=\lambda_{3} x_{1}(t)+\lambda_{4} x_{2}(t)-\left(\mu_{1}+\mu_{2}\right) x_{3}(t)
\end{array}\right\},
$$

where $x_{1}(t)$ denotes the probability of being in the state $S_{1}$ at time $t, x_{2}(t)$ denotes the probability of being in the state $S_{2}$ at time $t$ and $x_{3}(t)$ denotes the probability of being in the state $S_{3}$ at time $t$, respectively.

Conducting the Laplace transformation on Eq. (3.1) and Eq. (3.2), then we have

$$
\begin{aligned}
& s x_{1}(s)=-\left(\lambda_{2}+\lambda_{3}\right) x_{1}(s)+\lambda_{1} x_{2}(s)+\mu_{1} x_{3}(s)+x_{1}(0) \\
& \left.s x_{2}(s)=\lambda_{2} x_{1}(s)-\left(\lambda_{1}+\lambda_{4}\right) x_{2}(s)+\mu_{2} x_{3}(s)+x_{2}(0)\right\} \text {. } \\
& x_{1}(s)+x_{2}(s)+x_{3}(s)=\frac{1}{s}
\end{aligned}
$$

Firstly, we define state $S_{1}$ and state $S_{2}$ as the normal state, and the availability of the system denotes the probability of being in the normal state at time $t$. Thus, we have

$$
A(t)=x_{1}(t)+x_{2}(t)
$$

where $A(t)$ denotes the availability of the system at time $t$.

Theorem 1. As the checking rate is constant, the steady-state availability of the system and the MTTF of the system is, respectively

$$
\begin{gathered}
A_{0}=\lim _{t \rightarrow \infty} A(t)=x_{1}(\infty)+x_{2}(\infty)=\frac{\mu_{1}\left(\lambda_{1}+\lambda_{2}+\lambda_{4}\right)+\mu_{2}\left(\lambda_{1}+\lambda_{2}+\lambda_{3}\right)}{\left(\lambda_{1}+\lambda_{2}\right)\left(\mu_{1}+\mu_{2}\right)+\lambda_{3}\left(\lambda_{1}+\mu_{2}\right)+\lambda_{4}\left(\lambda_{2}+\mu_{1}\right)+\lambda_{3} \lambda_{4}}, \\
\operatorname{MTTF}=\frac{\left(\lambda_{1}+\lambda_{2}+\lambda_{4}\right) x_{1}(0)+\left(\lambda_{1}+\lambda_{2}+\lambda_{3}\right) x_{2}(0)}{\lambda_{1} \lambda_{3}+\lambda_{2} \lambda_{4}+\lambda_{3} \lambda_{4}} .
\end{gathered}
$$

Proof. From Eq. (3.3), we can obtain 


$$
\operatorname{sx}^{*}(s)=\boldsymbol{A}_{1} \boldsymbol{x} \stackrel{*}{(s)}+\boldsymbol{b},
$$

where $\boldsymbol{x}(s)$ denotes the Laplace transformation of $\boldsymbol{x}(t)$,

$$
\boldsymbol{x}(s)=\left[\begin{array}{c}
x_{1}(s) \\
\dot{x_{2}}(s)
\end{array}\right], \boldsymbol{A}_{1}=\left[\begin{array}{ll}
a_{1} & a_{2} \\
a_{3} & a_{4}
\end{array}\right]=\left[\begin{array}{cc}
-\left(\lambda_{2}+\lambda_{3}+\mu_{1}\right) & \lambda_{1}-\mu_{1} \\
\lambda_{2}-\mu_{2} & -\left(\lambda_{1}+\lambda_{4}+\mu_{2}\right)
\end{array}\right], \boldsymbol{b}=\left[\begin{array}{c}
\frac{\mu_{1}}{s}+x_{1}(0) \\
\frac{\mu_{2}}{s}+x_{2}(0)
\end{array}\right] .
$$

To ensure that the system is asymptotically stable, $\boldsymbol{A}_{1}$ must match that $\left(\boldsymbol{I}-\boldsymbol{A}_{1}\right)$ is nonsingular matrix and $\left(\boldsymbol{I}+\boldsymbol{A}_{1}\right)\left(\boldsymbol{I}-\boldsymbol{A}_{1}\right)^{-1}$ is convergent matrix, $\boldsymbol{I}$ is unit matrix [12-13].

Then, we can obtain

$$
\begin{gathered}
\boldsymbol{x}(s)=\left[\begin{array}{l}
\frac{\left(s-a_{4}\right)\left(x_{1}(0)+\frac{\mu_{1}}{s}\right)+a_{2}\left(x_{2}(0)+\frac{\mu_{2}}{s}\right)}{\operatorname{det}\left|s \boldsymbol{I}-\boldsymbol{A}_{1}\right|} \\
\frac{a_{3}\left(x_{1}(0)+\frac{\mu_{1}}{s}\right)+\left(s-a_{1}\right)\left(x_{2}(0)+\frac{\mu_{2}}{s}\right)}{\operatorname{det}\left|s \boldsymbol{I}-\boldsymbol{A}_{1}\right|}
\end{array}\right], \\
\operatorname{det}\left|s \boldsymbol{I}-\boldsymbol{A}_{1}\right|=s^{2}-\left(a_{1}+a_{4}\right) s+a_{1} a_{4}-a_{2} a_{3} .
\end{gathered}
$$

According to Laplace transformation, and we have

$$
\boldsymbol{x}(\infty)=\lim _{s \rightarrow 0} s \boldsymbol{x}(s)
$$

Combining Eq. (3.8), Eq. (3.9) and Eq. (3.10), we can obtain

$$
\begin{aligned}
& x_{1}(\infty)=\frac{\mu_{1}\left(\lambda_{1}+\lambda_{4}\right)+\mu_{2} \lambda_{1}}{\left(\lambda_{1}+\lambda_{2}\right)\left(\mu_{1}+\mu_{2}\right)+\lambda_{3}\left(\lambda_{1}+\mu_{2}\right)+\lambda_{4}\left(\lambda_{2}+\mu_{1}\right)+\lambda_{3} \lambda_{4}}, \\
& x_{2}(\infty)=\frac{\mu_{1} \lambda_{2}+\mu_{2}\left(\lambda_{2}+\lambda_{3}\right)}{\left(\lambda_{1}+\lambda_{2}\right)\left(\mu_{1}+\mu_{2}\right)+\lambda_{3}\left(\lambda_{1}+\mu_{2}\right)+\lambda_{4}\left(\lambda_{2}+\mu_{1}\right)+\lambda_{3} \lambda_{4}} .
\end{aligned}
$$

Based on the above discussions, the steady-state availability of the system can be obtained, immediately.

Let state $S_{3}$ be the absorbing state, thus $x_{3}(t)=0$ from the beginning. And set $R(t)=x_{1}(t)+x_{2}(t), \mu_{1}=\mu_{2}=0$, where $R(t)$ denotes the reliability of the system. So, we have

$$
\mathrm{MTTF}=\int_{0}^{\infty} R(t) \mathrm{d} t=R(s)\left|s_{=0}=\left(x_{1}(s)+x_{2}(s)\right)\right| s=0 .
$$

Then, Eq. (3.6) can be obtained, immediately. 


\section{Control Strategy Design}

To make the dynamic behavior of the system achieve the expected steady-state aim, we may control the checking rate of the maintenance system, which are the inverse of mean time between checks.

According to Eq. (3.1) and Eq. (3.2), we have

$$
\left.\begin{array}{l}
\frac{\mathrm{d} x_{1}(t)}{\mathrm{d} t}=-\left(\lambda_{2}+v_{1}+\mu_{1}\right) x_{1}(t)+\left(\lambda_{1}-\mu_{1}\right) x_{2}(t)-u_{1}(t) x_{1}(t)+\mu_{1} \\
\frac{\mathrm{d} x_{2}(t)}{\mathrm{d} t}=\left(\lambda_{2}-\mu_{2}\right) x_{1}(t)-\left(\lambda_{1}+v_{2}+\mu_{2}\right) x_{2}(t)-u_{2}(t) x_{2}(t)+\mu_{2}
\end{array}\right\} .
$$

Writing Eq. (4.1) as the Matrix form, then we can obtain

$$
\frac{\mathrm{d} \boldsymbol{x}(t)}{\mathrm{d} t}=\boldsymbol{A} \boldsymbol{x}(t)-\boldsymbol{U}(t) \boldsymbol{x}(t)+\boldsymbol{\mu}=\boldsymbol{A} \boldsymbol{x}(t)-\boldsymbol{X}(t) \boldsymbol{u}(t)+\boldsymbol{\mu},
$$

where,

$$
\begin{gathered}
\boldsymbol{A}=\left[\begin{array}{cc}
-d_{1} & a \\
b & -d_{2}
\end{array}\right]=\left[\begin{array}{cc}
-\left(\lambda_{2}+v_{1}+\mu_{1}\right) & \lambda_{1}-\mu_{1} \\
\lambda_{2}-\mu_{2} & -\left(\lambda_{1}+v_{2}+\mu_{2}\right)
\end{array}\right], \boldsymbol{x}(t)=\left[\begin{array}{l}
x_{1}(t) \\
x_{2}(t)
\end{array}\right], \\
\boldsymbol{X}(t)=\left[\begin{array}{cc}
x_{1}(t) & 0 \\
0 & x_{2}(t)
\end{array}\right], \boldsymbol{u}(t)=\left[\begin{array}{l}
u_{1}(t) \\
u_{2}(t)
\end{array}\right], \boldsymbol{U}(t)=\left[\begin{array}{cc}
u_{1}(t) & 0 \\
0 & u_{2}(t)
\end{array}\right], \boldsymbol{\mu}=\left[\begin{array}{l}
\mu_{1} \\
\mu_{2}
\end{array}\right] .
\end{gathered}
$$

Projecting the system (3.1) onto a plane of $x_{1}$ and $x_{2}$, then we have

$$
x_{1}(t)+x_{2}(t) \leq 1, t \geq 0 .
$$

As $x_{1}$ or $x_{2}$ tends to zero, to ensure the boundness of $\boldsymbol{u}(t)$, where $\boldsymbol{u}(t)=\left[\begin{array}{ll}u_{1}(t) & u_{2}(t)\end{array}\right]^{\mathrm{T}}$, we divide the area of $x_{1}$ and $x_{2}$ into four parts which are denoted respectively with (1) (4) as shown in Figure 2, where $0<\rho<1$.

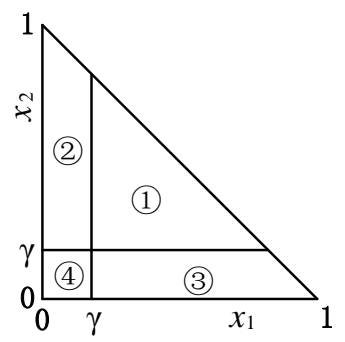

Figure 2. Valid Area of Control Variable

Theorem 2. In the area (1, $\rho \leq x_{1} \leq 1-\rho, \rho \leq x_{2} \leq 1-\rho$, and $x_{1}+x_{2} \leq 1$. We do the control law for $\boldsymbol{u}(t)$ below.

$$
\boldsymbol{u}(t)=\left[\begin{array}{l}
u_{1}(t) \\
u_{2}(t)
\end{array}\right]=[\boldsymbol{X}(t)]^{-1}\left[\left(\boldsymbol{A}+\boldsymbol{K}_{\mathrm{ml}}\right) \boldsymbol{x}(t)+\boldsymbol{\mu}-\boldsymbol{K}_{\mathrm{m} 1} \overline{\boldsymbol{x}}_{\mathrm{m} 1}\right]
$$


where $\overline{\boldsymbol{x}}_{\mathrm{m} 1}=\left[\begin{array}{l}\bar{x}_{1 \mathrm{~m} 1} \\ \bar{x}_{2 \mathrm{~m} 1}\end{array}\right], \boldsymbol{K}_{\mathrm{ml}}=\left[\begin{array}{ll}k_{11} & k_{12} \\ k_{13} & k_{14}\end{array}\right], \overline{\boldsymbol{x}}_{\mathrm{m} 1}$ is the expected steady-state probability, $\boldsymbol{K}_{\mathrm{ml}}$ matches $\left(\boldsymbol{I}+\boldsymbol{K}_{\mathrm{ml}}\right)$ is nonsingular matrix and $\left(\boldsymbol{I}-\boldsymbol{K}_{\mathrm{ml}}\right)\left(\boldsymbol{I}+\boldsymbol{K}_{\mathrm{ml}}\right)^{-1}$ is convergent matrix.

Under the action of the control law (4.4), the steady-state availability of the system becomes

$$
A_{0}=x_{1}(\infty)+x_{2}(\infty)=\bar{x}_{1 \mathrm{~m} 1}+\bar{x}_{2 \mathrm{ml}} .
$$

Proof. Known from Theorem 1, the desired state equation should possess the following form.

$$
\frac{\mathrm{d} \boldsymbol{x}(t)}{\mathrm{d} t}=\boldsymbol{A}_{\mathrm{m} \mathrm{l}} \boldsymbol{x}(t)+\boldsymbol{\mu}_{\mathrm{ml}}
$$

And so, the steady-state value of the system can be written as

$$
\boldsymbol{x}(\infty)=\overline{\boldsymbol{x}}=-\left[\boldsymbol{A}_{\mathrm{ml}}\right]^{-1} \boldsymbol{\mu}_{\mathrm{ml}}=\overline{\boldsymbol{x}}_{\mathrm{ml}}=\left(\begin{array}{ll}
\bar{x}_{\mathrm{ml} 1} & \bar{x}_{2 \mathrm{ml}}
\end{array}\right)^{\mathrm{T}} .
$$

Let $\boldsymbol{A}_{\mathrm{ml}}=-\boldsymbol{K}_{\mathrm{ml}}, \boldsymbol{\mu}_{\mathrm{m} 1}=\boldsymbol{K}_{\mathrm{m} 1} \overline{\boldsymbol{x}}_{\mathrm{m} 1}$ and combining Eq. (4.2) and Eq. (4.6), we can get Eq. (4.4), and Eq. (4.5) by combining Eq. (4.2), Eq. (4.4) and Eq. (4.6). To ensure that the system is asymptotically stable, $\boldsymbol{K}_{\mathrm{m} 1}$ must match that $\left(\boldsymbol{I}+\boldsymbol{K}_{\mathrm{ml}}\right)$ is nonsingular matrix and $\left(\boldsymbol{I}-\boldsymbol{K}_{\mathrm{m} 1}\right)\left(\boldsymbol{I}+\boldsymbol{K}_{\mathrm{m} 1)^{-1}}\right.$ is convergent matrix ${ }^{[12-13]}$.

End.

In the area 2, in order to ensure that $u_{1}(t)$ is bounded, we let $u_{1}(t)$ be constant, but $u_{2}(t)$ is variable whose form is similar to the one as showed in Eq. (4.4). Thus, the control law for $\boldsymbol{u}(t)$ becomes

$$
\boldsymbol{u}(t)=\left[\begin{array}{l}
u_{1}(t) \\
u_{2}(t)
\end{array}\right]=\left[\begin{array}{c}
\frac{\mu_{1}+a \bar{x}_{2 \mathrm{~m} 2}}{\bar{x}_{1 \mathrm{~m} 2}}-d_{1} \\
\frac{\left(k_{23}+b\right) x_{1}(t)+\left(k_{24}-d_{2}\right) x_{2}(t)+\left(\mu_{2}-k_{23} \bar{x}_{1 \mathrm{~m} 2}-k_{24} \bar{x}_{2 \mathrm{~m} 2}\right)}{x_{2}(t)}
\end{array}\right],
$$

where,

$$
\overline{\boldsymbol{x}}_{\mathrm{m} 2}=\left[\begin{array}{l}
\bar{x}_{1 \mathrm{~m} 2} \\
\bar{x}_{2 \mathrm{~m} 2}
\end{array}\right], \quad \boldsymbol{K}_{\mathrm{m} 2}=\left[\begin{array}{ll}
k_{21} & k_{22} \\
k_{23} & k_{24}
\end{array}\right]=\left[\begin{array}{cc}
\frac{\mu_{1}+a \bar{x}_{2 \mathrm{~m} 2}}{\bar{x}_{1 \mathrm{~m} 2}} & -a \\
k_{23} & k_{24}
\end{array}\right],
$$

$\overline{\boldsymbol{x}}_{\mathrm{m} 2}$ denotes the expected steady-state probability when the system starts from the area (2). Under the action of the control law (4.7), the dynamic behavior of the system becomes

$$
\frac{\mathrm{d} \boldsymbol{x}(t)}{\mathrm{d} t}=\boldsymbol{A}_{\mathrm{m} 2} \boldsymbol{x}(t)+\boldsymbol{\mu}_{\mathrm{m} 2},
$$

where $\boldsymbol{A}_{\mathrm{m} 2}=-\boldsymbol{K}_{\mathrm{m} 2}, \boldsymbol{\mu}_{\mathrm{m} 2}=\boldsymbol{K}_{\mathrm{m} 2} \overline{\boldsymbol{x}}_{\mathrm{m} 2}=\left[\begin{array}{c}\mu_{1} \\ k_{23} \overline{\boldsymbol{x}}_{\mathrm{lm} 2}+k_{24} \overline{\boldsymbol{x}}_{2 \mathrm{~m} 2}\end{array}\right]$. In order to ensure that the system is asymptotically stable, $\boldsymbol{A}_{\mathrm{m} 2}$ matches that $\left(\boldsymbol{I}-\boldsymbol{A}_{\mathrm{m} 2}\right)$ is nonsingular matrix and $\left(\boldsymbol{I}+\boldsymbol{A}_{\mathrm{m} 2}\right)\left(\boldsymbol{I}-\boldsymbol{A}_{\mathrm{m} 2}\right)^{-1}$ is convergent matrix.

Thus, the steady-state value of the system becomes 


$$
\boldsymbol{x}(\infty)=\overline{\boldsymbol{x}}=-\left[\boldsymbol{A}_{\mathrm{m} 2}\right]^{-1} \boldsymbol{\mu}_{\mathrm{m} 2}=\overline{\boldsymbol{x}}_{\mathrm{m} 2}=\left(\begin{array}{ll}
\bar{x}_{1 \mathrm{~m} 2} & \bar{x}_{2 \mathrm{~m} 2}
\end{array}\right)^{\mathrm{T}} .
$$

In the same way, In the area (3), we set $u_{2}(t)$ as constant, but $u_{1}(t)$ is variable whose form is similar to the one as showed in Eq. (4.4). Thus, the control law for $\boldsymbol{u}(t)$ becomes

$$
\boldsymbol{u}(t)=\left[\begin{array}{l}
u_{1}(t) \\
u_{2}(t)
\end{array}\right]=\left[\begin{array}{c}
\frac{\left(k_{31}-d_{1}\right) x_{1}(t)+\left(k_{32}+a\right) x_{2}(t)+\left(\mu_{1}-k_{31} \bar{x}_{1 \mathrm{~m} 3}-k_{32} \bar{x}_{2 \mathrm{~m} 3}\right)}{x_{1}(t)} \\
\frac{\mu_{2}+b \bar{x}_{1 \mathrm{~m} 3}}{\bar{x}_{2 \mathrm{~m} 3}}-d_{2}
\end{array}\right],
$$

where,

$$
\overline{\boldsymbol{x}}_{\mathrm{m} 3}=\left[\begin{array}{l}
\bar{x}_{1 \mathrm{~m} 3} \\
\bar{x}_{2 \mathrm{~m} 3}
\end{array}\right], \boldsymbol{K}_{\mathrm{m} 3}=\left[\begin{array}{ll}
k_{31} & k_{32} \\
k_{33} & k_{34}
\end{array}\right]=\left[\begin{array}{cc}
k_{31} & k_{32} \\
-b & \frac{\mu_{2}+b \bar{x}_{1 \mathrm{~m} 3}}{\bar{x}_{2 \mathrm{~m} 3}}
\end{array}\right],
$$

$\overline{\boldsymbol{x}}_{\mathrm{m} 3}$ denotes the expected steady-state probability when the system starts from the area (3) Under the action of the control law (4.9), the dynamic behavior of the system becomes

$$
\frac{\mathrm{d} \boldsymbol{x}(t)}{\mathrm{d} t}=\boldsymbol{A}_{\mathrm{m} 3} \boldsymbol{x}(t)+\boldsymbol{\mu}_{\mathrm{m} 3}
$$

where $\boldsymbol{A}_{\mathrm{m} 3}=-\boldsymbol{K}_{\mathrm{m} 3}, \boldsymbol{\mu}_{\mathrm{m} 3}=\boldsymbol{K}_{\mathrm{m} 3} \overline{\boldsymbol{x}}_{\mathrm{m} 3}=\left[\begin{array}{c}k_{31} \bar{x}_{1 \mathrm{~m} 3}+k_{32} \bar{x}_{2 \mathrm{~m} 3} \\ \mu_{2}\end{array}\right]$. In order to ensure that the system is asymptotically stable, $\boldsymbol{A}_{\mathrm{m} 3}$ matches that $\left(\boldsymbol{I}-\boldsymbol{A}_{\mathrm{m} 3}\right)$ is nonsingular matrix and $\left(\boldsymbol{I}+\boldsymbol{A}_{\mathrm{m} 3}\right)\left(\boldsymbol{I}-\boldsymbol{A}_{\mathrm{m} 3}\right)^{-1}$ is convergent matrix.

Thus, the steady-state value of the system becomes

$$
\boldsymbol{x}(\infty)=\overline{\boldsymbol{x}}=-\left[\boldsymbol{A}_{\mathrm{m} 3}\right]^{-1} \boldsymbol{\mu}_{\mathrm{m} 3}=\overline{\boldsymbol{x}}_{\mathrm{m} 3}=\left(\begin{array}{ll}
\bar{x}_{1 \mathrm{~m} 3} & \bar{x}_{2 \mathrm{~m} 3}
\end{array}\right)^{\mathrm{T}} .
$$

In the same way, In the area (4, we set the control law for $\boldsymbol{u}(t)$ as

$$
\boldsymbol{u}(t)=\left[\begin{array}{l}
u_{1}(t) \\
u_{2}(t)
\end{array}\right]=\left[\begin{array}{l}
\frac{\mu_{1}+a \bar{x}_{2 \mathrm{~m} 4}}{\bar{x}_{1 \mathrm{~m} 4}}-d_{1} \\
\frac{\mu_{2}+b \bar{x}_{1 \mathrm{~m} 4}}{\bar{x}_{2 \mathrm{~m} 4}}-d_{2}
\end{array}\right]
$$

where,

$$
\overline{\boldsymbol{x}}_{\mathrm{m} 4}=\left[\begin{array}{c}
\bar{x}_{1 \mathrm{~m} 4} \\
\bar{x}_{2 \mathrm{~m} 4}
\end{array}\right], \boldsymbol{K}_{\mathrm{m} 4}=\left[\begin{array}{ll}
k_{41} & k_{42} \\
k_{43} & k_{44}
\end{array}\right]=\left[\begin{array}{cc}
\frac{\mu_{1}+a \bar{x}_{2 \mathrm{~m} 4}}{\bar{x}_{1 \mathrm{~m} 4}} & -a \\
-b & \frac{\mu_{2}+b \bar{x}_{1 \mathrm{~m} 4}}{\bar{x}_{2 \mathrm{~m} 4}}
\end{array}\right],
$$

$\overline{\boldsymbol{x}}_{\mathrm{m} 4}$ denotes the expected steady-state probability when the system starts from the area (4). Under the action of the control law (4.11), the dynamic behaviour of the system becomes

$$
\frac{\mathrm{d} \boldsymbol{x}(t)}{\mathrm{d} t}=\boldsymbol{A}_{\mathrm{m} 4} \boldsymbol{x}(t)+\boldsymbol{\mu}_{\mathrm{m} 4},
$$


where $\boldsymbol{A}_{\mathrm{m} 4}=-\boldsymbol{K}_{\mathrm{m} 4}$ and $\boldsymbol{\mu}_{\mathrm{m} 4}=\boldsymbol{K}_{\mathrm{m} 4} \overline{\boldsymbol{x}}_{\mathrm{m} 4}=\left[\begin{array}{l}\mu_{1} \\ \mu_{2}\end{array}\right]$. In order to ensure that the system (4.12) is asymptotically stable, $\boldsymbol{A}_{\mathrm{m} 4}$ matches that $\left(\boldsymbol{I}-\boldsymbol{A}_{\mathrm{m} 4}\right)$ is nonsingular matrix and $\left(\boldsymbol{I}+\boldsymbol{A}_{\mathrm{m} 4)}\right)\left(\boldsymbol{I}-\boldsymbol{A}_{\mathrm{m} 4}\right)^{-1}$ is convergent matrix.

Thus, the steady-state value of the system becomes

$$
\boldsymbol{x}(\infty)=\overline{\boldsymbol{x}}=-\left[\boldsymbol{A}_{\mathrm{m} 4}\right]^{-1} \boldsymbol{\mu}_{\mathrm{m} 4}=\overline{\boldsymbol{x}}_{\mathrm{m} 4}=\left(\begin{array}{ll}
\bar{x}_{1 \mathrm{~m} 4} & \left.\bar{x}_{2 \mathrm{~m} 4}\right)^{\mathrm{T}} .
\end{array}\right.
$$

Under the action of the control laws (4.4), (4.7), (4.9) and (4.11), the form of the motion equation of the system is as follows.

$$
\frac{\mathrm{d} \boldsymbol{x}(t)}{\mathrm{d} t}=\boldsymbol{A}_{\mathrm{m} i \boldsymbol{x}}(t)+\boldsymbol{\mu}_{\mathrm{m} i},(i=1,2,3,4) .
$$

Then, solving Eq. (4.13), we get

$$
\boldsymbol{x}(t)=\exp \left(\boldsymbol{A}_{\mathrm{mi} i}\right) \boldsymbol{x}(0)+\int_{0}^{t} \exp \left(\boldsymbol{A}_{\mathrm{mi}}(t-\tau)\right) \mathrm{d} \tau \cdot \boldsymbol{\mu}_{\mathrm{m} i},(i=1,2,3,4) .
$$

According to matrix $\boldsymbol{A}_{\mathrm{m} i}$, we can know that the system is asymptotically stable. So, we have

$$
\boldsymbol{x}(t)=\exp \left(\boldsymbol{A}_{\mathrm{mi}} t\right)\left(\boldsymbol{x}(0)+\boldsymbol{A}_{\mathrm{m} i}{ }^{-1} \boldsymbol{\mu}_{\mathrm{m} i}\right)-\boldsymbol{A}_{\mathrm{m} i}{ }^{-1} \boldsymbol{\mu}_{\mathrm{m} i},(i=1,2,3,4) .
$$

Thus, the steady-state value of the system is

$$
\boldsymbol{x}(\infty)=-\boldsymbol{A}_{\mathrm{m} i}{ }^{-1} \boldsymbol{\mu}_{\mathrm{m} i}=\overline{\boldsymbol{x}}_{\mathrm{mi}},(i=1,2,3,4) .
$$

Then, we can obtain

$$
\boldsymbol{x}(t)=\exp \left(\boldsymbol{A}_{\mathrm{mi} i} t\right)\left(\boldsymbol{x}(0)-\overline{\boldsymbol{x}}_{\mathrm{m} i}\right)+\overline{\boldsymbol{x}}_{\mathrm{mi}},(i=1,2,3,4) .
$$

Under the condition that the initial value $\boldsymbol{x}(0)$ and the expected steady-state value $\overline{\boldsymbol{x}}_{\mathrm{m}}$ are given out, it is quite obvious that the motion equation of the system is only related to $\exp \left(\boldsymbol{A}_{\mathrm{m} i t}\right)$. So, we use the method of resolvent matrix to solve $\exp \left(\boldsymbol{A}_{\mathrm{mi}} t\right)$ [12]. Thus, we have

$$
\exp \left(\boldsymbol{A}_{\mathrm{m} i} t\right)=L^{-1}\left(\left(s \boldsymbol{I}-\boldsymbol{A}_{\mathrm{m} i}\right)^{-1}\right),
$$

where $L^{-1}(\bullet)$ denotes the inverse Laplace transformation.

Firstly, we conduct the following definitions.

$$
\begin{gathered}
p_{1}(s)=s^{2}+s\left(k_{11}+k_{14}\right)+k_{11} k_{14}-k_{12} k_{13} . \\
p_{2}(s)=s^{2}+s\left(\frac{\mu_{1}+a \bar{x}_{2 \mathrm{~m} 2}}{\bar{x}_{1 \mathrm{~m} 2}}+k_{24}\right)+\frac{\left(\mu_{1}+a \bar{x}_{2 \mathrm{~m} 2}\right) k_{24}}{\bar{x}_{1 \mathrm{~m} 2}}+a k_{23} . \\
p_{3}(s)=s^{2}+s\left(k_{31}+\frac{\mu_{2}+b \bar{x}_{\mathrm{Im} 3}}{\bar{x}_{2 \mathrm{~m} 3}}\right)+\frac{\left(\mu_{2}+b \bar{x}_{\mathrm{Im} 3}\right) k_{31}}{\bar{x}_{2 \mathrm{~m} 3}}+b k_{32} .
\end{gathered}
$$




$$
\begin{gathered}
p_{4}(s)=s^{2}+s\left(\frac{\mu_{1}+a \bar{x}_{2 \mathrm{~m} 4}}{\bar{x}_{1 \mathrm{~m} 4}}+\frac{\mu_{2}+b \bar{x}_{1 \mathrm{~m} 4}}{\bar{x}_{2 \mathrm{~m} 4}}\right)+\frac{\left(\mu_{1}+a \bar{x}_{2 \mathrm{~m} 4}\right)}{\bar{x}_{1 \mathrm{~m} 4}} \frac{\left(\mu_{2}+b \bar{x}_{1 \mathrm{~m} 4}\right)}{\bar{x}_{2 \mathrm{~m} 4}}-a b . \\
q_{1}(s)=s+\frac{\mu_{1}+a \bar{x}_{2 \mathrm{~m} 2}}{\bar{x}_{1 \mathrm{~m} 2}}, q_{2}(s)=s+\frac{\mu_{2}+b \bar{x}_{1 \mathrm{~m} 3}}{\bar{x}_{2 \mathrm{~m} 3}} . \\
q_{3}(s)=s+\frac{\mu_{2}+b \bar{x}_{1 \mathrm{~m} 4}}{\bar{x}_{2 \mathrm{~m} 4}}, q_{4}(s)=s+\frac{\mu_{1}+a \bar{x}_{2 \mathrm{~m} 4}}{\bar{x}_{1 \mathrm{~m} 4}} .
\end{gathered}
$$

And then, we can obtain

$$
\begin{aligned}
& \exp \left(\boldsymbol{A}_{\mathrm{m} 1} t\right)=\left[\begin{array}{ll}
f_{11}(t) & f_{12}(t) \\
f_{13}(t) & f_{14}(t)
\end{array}\right]=L^{-1}\left[\begin{array}{cc}
\frac{s+k_{14}}{p_{1}(s)} & \frac{-k_{12}}{p_{1}(s)} \\
\frac{-k_{13}}{p_{1}(s)} & \frac{s+k_{11}}{p_{1}(s)}
\end{array}\right] \\
& \exp \left(\boldsymbol{A}_{\mathrm{m} 2} t\right)=\left[\begin{array}{ll}
f_{21}(t) & f_{22}(t) \\
f_{23}(t) & f_{24}(t)
\end{array}\right]=L^{-1}\left[\begin{array}{cc}
\frac{s+k_{24}}{p_{2}(s)} & \frac{a}{p_{2}(s)} \\
\frac{-k_{23}}{p_{2}(s)} & \frac{q_{1}(s)}{p_{2}(s)}
\end{array}\right] . \\
& \exp \left(\boldsymbol{A}_{\mathrm{m} 3} t\right)=\left[\begin{array}{ll}
f_{31}(t) & f_{32}(t) \\
f_{33}(t) & f_{34}(t)
\end{array}\right]=L^{-1}\left[\begin{array}{cc}
\frac{q_{2}(s)}{p_{3}(s)} & \frac{-k_{32}}{p_{3}(s)} \\
\frac{b}{p_{3}(s)} & \frac{s+k_{31}}{p_{3}(s)}
\end{array}\right] . \\
& \exp \left(\boldsymbol{A}_{\mathrm{m} 4 t} t\right)=\left[\begin{array}{ll}
f_{41}(t) & f_{42}(t) \\
f_{43}(t) & f_{44}(t)
\end{array}\right]=L^{-1}\left[\begin{array}{cc}
\frac{q_{3}(s)}{p_{4}(s)} & \frac{a}{p_{4}(s)} \\
\frac{b}{p_{4}(s)} & \frac{q_{4}(s)}{p_{4}(s)}
\end{array}\right] .
\end{aligned}
$$

Based on the above discussions, under the condition that the initial value $x(0)$ and the expected steady-state value $\overline{\boldsymbol{x}}_{\mathrm{m} i}$ are given out, we can get the motion equation $\boldsymbol{x}(t)$ of the system by combining $\boldsymbol{x}(\infty)=-\boldsymbol{A}_{\mathrm{m} i}{ }^{-1} \boldsymbol{\mu}_{\mathrm{m} i}=\overline{\boldsymbol{x}}_{\mathrm{m} i}$ and $\exp \left(\boldsymbol{A}_{\mathrm{m} i} t\right)$.

\section{Probability Estimation}

Definition 1. According to literature [14], the probability of the system being in the state $S_{i}$ at time $t$ can be expressed by

$$
x_{i}(t)=\frac{n_{i}(t)}{n(t)}
$$

where $n_{i}(t)$ represents the number of the device being in the state $S_{i}$ at time $t$, and $n(t)$ represents the total number of the same device being in the state $S_{i}$ at the same time. Note that

$$
\hat{x}_{i}(t)=x_{i}(t)+\hat{\delta}_{i}(t)
$$


where $\hat{\delta}_{i}(t)$ expresses the error between $\hat{x}_{i}(t)$ and $x_{i}(t)$, which is a bounded random variable. For $n(t)$ large enough, it follows normal distribution $N\left(0, \sigma_{x i}\right)$.

And then

$$
E\left(x_{i}(t)\right)=x_{i}(t)
$$

The significance of the above formula lies in that $x_{i}(t)$ possesses same evolution law with $\boldsymbol{x}(t)$ even though it is a probability statistics variable. As a fact, due to initial values and system arguments given, it is necessary that there exists one connection between both.

In order to quote some lemmas, we conduct some definitions as follows. $\|\boldsymbol{A}\|:$ It denotes the Euclidean norm of the matrix $\boldsymbol{A}$.

$|A|:$ It denotes the matrix that is made up of the elements that are the modulus value of every element in the matrix $\boldsymbol{A}$.

$\boldsymbol{R e}(\square)$ : It denotes the real part.

$\operatorname{diag}(\mathrm{g})$ : It denotes the diagonal matrix.

$\boldsymbol{A} \leq \boldsymbol{B}$ : It denotes $a_{i j} \leq b_{i j}(\forall i, j)$, where $\boldsymbol{A}=\left(a_{i j}\right)$ and $\boldsymbol{B}=\left(b_{i j}\right)$.

$\operatorname{cond}(\boldsymbol{A})$ : It denotes condition number of the matrix $\boldsymbol{A}$ and $\operatorname{cond}(\boldsymbol{A})=\|\boldsymbol{A}\|\left\|\boldsymbol{A}^{-1}\right\|$.

As the checking rate is constant, we have

$$
\frac{\mathrm{d} \boldsymbol{x}(t)}{\mathrm{d} t}=\boldsymbol{A} \boldsymbol{x}(t)-\boldsymbol{U} \boldsymbol{x}(t)+\boldsymbol{\mu}
$$

where $U$ is determined by Eq. (4.11).

Let $\hat{\boldsymbol{x}}(t)$ denote an estimate value of $\boldsymbol{x}(t)$ determined by system (5.4), and then we can obtain

$$
\frac{\mathrm{d} \hat{\boldsymbol{x}}(t)}{\mathrm{d} t}=\boldsymbol{A} \hat{\boldsymbol{x}}(t)-\boldsymbol{U} \hat{\boldsymbol{x}}(t)+\boldsymbol{\mu}
$$

So, we have

$$
\hat{\boldsymbol{\delta}}(t)=\hat{\boldsymbol{x}}(t)-\boldsymbol{x}(t)
$$

where $\hat{\boldsymbol{\delta}}(t)$ expresses the error between $\hat{\boldsymbol{x}}(t)$ and $\boldsymbol{x}(t)$.

With initial conditions satisfying

$$
\hat{\boldsymbol{x}}(0)=\hat{x}(0)=\boldsymbol{x}(0)+\hat{\boldsymbol{\delta}}(0)=\boldsymbol{x}(0)+\hat{\boldsymbol{\delta}}(0)
$$

According to Eq. (5.6), we can obtain

$$
\begin{aligned}
\frac{\mathrm{d} \hat{\boldsymbol{\delta}}(t)}{\mathrm{d} t} & =\frac{\mathrm{d} \hat{\boldsymbol{x}}(t)}{\mathrm{d} t}-\frac{\mathrm{d} \boldsymbol{x}(t)}{\mathrm{d} t}=\boldsymbol{A} \hat{\boldsymbol{x}}(t)-\boldsymbol{U} \hat{\boldsymbol{x}}(t)+\boldsymbol{\mu}-(\boldsymbol{A x}(t)-\boldsymbol{U} \boldsymbol{x}(t)+\boldsymbol{\mu}) \\
& =\boldsymbol{A}(\hat{\boldsymbol{x}}(t)-\boldsymbol{x}(t))-\boldsymbol{U}(\hat{\boldsymbol{x}}(t)-\boldsymbol{x}(t))=(\boldsymbol{A}-\boldsymbol{U}) \hat{\boldsymbol{\delta}}(t)
\end{aligned}
$$

Since $(\boldsymbol{A}-\boldsymbol{U})$ is a stable matrix, so, the system (5.8) is asymptotically stable. Thus, we have

$$
\hat{\boldsymbol{\delta}}(\infty)=\lim _{t \rightarrow \infty} \hat{\boldsymbol{\delta}}(t)=0
$$


And then, we can obtain

$$
\boldsymbol{x}(\infty)=\lim _{t \rightarrow \infty} \boldsymbol{x}(t)=\lim _{t \rightarrow \infty}(\hat{\boldsymbol{x}}(t)-\hat{\boldsymbol{\delta}}(t))=\hat{\boldsymbol{x}}(\infty)-\hat{\boldsymbol{\delta}}(\infty)=\hat{\boldsymbol{x}}(\infty)
$$

According to Eq. (5.10), we can know that the steady-state value of the estimation system and the steady-state value of the actual system are equal.

From Eq. (5.8) we can obtain

$$
\hat{\boldsymbol{\delta}}(t)=\exp ((\boldsymbol{A}-\boldsymbol{U}) t) \hat{\boldsymbol{\delta}}(0)=\exp (-\boldsymbol{\beta} t) \hat{\boldsymbol{\delta}}(0)
$$

where $\boldsymbol{\beta}=\boldsymbol{U}-\boldsymbol{A}$.

Theorem 3. In the area (4), under the action of the control law (4.11), the state probability $\boldsymbol{x}(t)$ of the system satisfies

$$
\left\|\boldsymbol{x}(t)-\overline{\boldsymbol{x}}_{\mathrm{m} 4}\right\| \leq \exp \left(-\alpha_{\mathrm{m} 4} t\right) \operatorname{cond}\left(\boldsymbol{T}_{\mathrm{m} 4}\right)\left(\|\hat{\boldsymbol{\delta}}(0)\|+\left\|\boldsymbol{x}(0)-\overline{\boldsymbol{x}}_{\mathrm{m} 4}\right\|\right)+\exp \left(-\alpha_{\mathrm{A}} t\right) \operatorname{cond}\left(\boldsymbol{T}_{\mathrm{A}}\right)\|\hat{\boldsymbol{\delta}}(0)\|
$$

where

$$
\begin{gathered}
\boldsymbol{T}_{\mathrm{m} 4}^{-1} \boldsymbol{A}_{\mathrm{m} 4} \boldsymbol{T}_{\mathrm{m} 4}=\boldsymbol{J}_{\mathrm{m} 4}=\operatorname{diag}\left(\lambda_{1}, \lambda_{2}\right) \\
\boldsymbol{T}_{\mathrm{A}}^{-1} \boldsymbol{A}_{\mathrm{1}} \boldsymbol{T}_{\mathrm{A}}=\boldsymbol{J}_{\mathrm{A}}=\operatorname{diag}\left(\lambda_{1}, \lambda_{2}\right) \\
\alpha_{\mathrm{m} 4}=-\max \left\{\operatorname{Re} \lambda_{i}\left(\boldsymbol{A}_{\mathrm{m} 4}\right)\right\}, \quad i=1,2 \\
\alpha_{\mathrm{A}}=-\max \left\{\operatorname{Re} \lambda_{i}\left(\boldsymbol{A}_{1}\right)\right\}, \quad i=1,2
\end{gathered}
$$

where $\boldsymbol{A}_{1}=\boldsymbol{A}-\boldsymbol{U}$.

Proof. Under the action of control law (4.11), Eq. (5.5) will become the following form.

$$
\frac{\mathrm{d} \hat{\boldsymbol{x}}(t)}{\mathrm{d} t}=\boldsymbol{A}_{\mathrm{m} 4} \hat{\boldsymbol{x}}(t)+\boldsymbol{\mu}_{\mathrm{m} 4}
$$

Then, we Let

$$
\hat{\boldsymbol{x}}(t)=\boldsymbol{T}_{\mathrm{m} 4} \overline{\boldsymbol{x}}(t)
$$

where

$$
\boldsymbol{T}_{\mathrm{m} 4}{ }^{-1} \boldsymbol{A}_{\mathrm{m} 4} \boldsymbol{T}_{\mathrm{m} 4}=\boldsymbol{J}_{\mathrm{m} 4}=\operatorname{diag}\left(\lambda_{1}, \lambda_{2}\right)
$$

And then, we let

$$
\alpha_{\mathrm{m} 4}=-\max \left\{\operatorname{Re} \lambda_{i}\left(\boldsymbol{A}_{\mathrm{m} 4}\right)\right\}, \quad i=1,2
$$

According Eq. (5.13) and Eq. (5.14), we can obtain

$$
\frac{\mathrm{d} \boldsymbol{T}_{\mathrm{m} 4} \overline{\boldsymbol{x}}(t)}{\mathrm{d} t}=\boldsymbol{A}_{\mathrm{m} 4} \boldsymbol{T}_{\mathrm{m} 4} \overline{\boldsymbol{x}}(t)+\boldsymbol{\mu}_{\mathrm{m} 4}
$$

So, we have

$$
\frac{\mathrm{d} \overline{\boldsymbol{x}}(t)}{\mathrm{d} t}=\boldsymbol{T}_{\mathrm{m} 4}{ }^{-1} \boldsymbol{A}_{\mathrm{m} 4} \boldsymbol{T}_{\mathrm{m} 4} \overline{\boldsymbol{x}}(t)+\boldsymbol{T}_{\mathrm{m} 4}{ }^{-1} \boldsymbol{\mu}_{\mathrm{m} 4}=\boldsymbol{J}_{\mathrm{m} 4} \overline{\boldsymbol{x}}(t)+\boldsymbol{T}_{\mathrm{m} 4}{ }^{-1} \boldsymbol{\mu}_{\mathrm{m} 4}
$$


Then, solving Eq. (5.16), we get

$$
\begin{aligned}
\overline{\boldsymbol{x}}(t) & =\exp \left(\boldsymbol{J}_{\mathrm{m} 4 t}\right) \overline{\boldsymbol{x}}(0)+\int_{0}^{t} \exp \left(\boldsymbol{J}_{\mathrm{m} 4}(t-\tau)\right) \mathrm{d} \tau \cdot \boldsymbol{T}_{\mathrm{m} 4}{ }^{-1} \boldsymbol{\mu}_{\mathrm{m} 4} . \\
& =\exp \left(\boldsymbol{J}_{\mathrm{m} 4 t}\right)(\overline{\boldsymbol{x}}(0)-\overline{\boldsymbol{x}}(\infty))+\overline{\boldsymbol{x}}(\infty)
\end{aligned}
$$

where $\overline{\boldsymbol{x}}(0)=\boldsymbol{T}_{\mathrm{m} 4}{ }^{-1} \hat{\boldsymbol{x}}(0)$ and $\overline{\boldsymbol{x}}(\infty)=\boldsymbol{T}_{\mathrm{m} 4}{ }^{-1} \hat{\boldsymbol{x}}(\infty)=-\boldsymbol{J}_{\mathrm{m} 4}{ }^{-1} \boldsymbol{T}_{\mathrm{m} 4}{ }^{-1} \boldsymbol{\mu}_{\mathrm{m} 4}=-\boldsymbol{T}_{\mathrm{m} 4}{ }^{-1} \boldsymbol{A}_{\mathrm{m} 4}{ }^{-1} \boldsymbol{\mu}_{\mathrm{m} 4}=\boldsymbol{T}_{\mathrm{m} 4}{ }^{-1} \overline{\boldsymbol{x}}_{\mathrm{m} 4}$.

So, we can obtain

$$
\overline{\boldsymbol{x}}(t)-\overline{\boldsymbol{x}}(\infty)=\exp \left(\boldsymbol{J}_{\mathrm{m} 4 t}\right)(\overline{\boldsymbol{x}}(0)-\overline{\boldsymbol{x}}(\infty))
$$

Based on the above analysis and literature [15], we have

$$
\begin{aligned}
\|\overline{\boldsymbol{x}}(t)-\overline{\boldsymbol{x}}(\infty)\| & =\left\|\exp \left(\boldsymbol{J}_{\mathrm{m} 4 t}\right)(\overline{\boldsymbol{x}}(0)-\overline{\boldsymbol{x}}(\infty))\right\| \leq\left\|\exp \left(\boldsymbol{J}_{\mathrm{m} 4 t}\right)\right\|\|\overline{\boldsymbol{x}}(0)-\overline{\boldsymbol{x}}(\infty)\| \\
& \leq\left\|\exp \left(\operatorname{Re} \boldsymbol{J}_{\mathrm{m} 4 t}\right)\right\|\|\overline{\boldsymbol{x}}(0)-\overline{\boldsymbol{x}}(\infty)\| \leq \exp \left(-\alpha_{\mathrm{m} 4 t}\right)\|\overline{\boldsymbol{x}}(0)-\overline{\boldsymbol{x}}(\infty)\|
\end{aligned}
$$

Then, we let

$$
\frac{\mathrm{d} \hat{\boldsymbol{\delta}}(t)}{\mathrm{d} t}=(\boldsymbol{A}-\boldsymbol{U}) \hat{\boldsymbol{\delta}}(t)=\boldsymbol{A} \boldsymbol{1} \hat{\boldsymbol{\delta}}(t)
$$

And then, we Let

$$
\hat{\boldsymbol{\delta}}(t)=\boldsymbol{T}_{\mathrm{A}} \overline{\boldsymbol{\delta}}(t)
$$

where

$$
\boldsymbol{T}_{\mathrm{A}}^{-1} \boldsymbol{A}_{1} \boldsymbol{T}_{\mathrm{A}}=\boldsymbol{J}_{\mathrm{A}}=\operatorname{diag}\left(\lambda_{1}, \lambda_{2}\right)
$$

And then, we let

$$
\alpha_{\mathrm{A}}=-\max \left(\operatorname{Re} \lambda_{i}\right), \quad i=1,2
$$

According Eq. (5.20) and Eq. (5.21), we can obtain

$$
\frac{\mathrm{d} \boldsymbol{T}_{\mathrm{A}} \overline{\boldsymbol{\delta}}(t)}{\mathrm{d} t}=\boldsymbol{A}_{1} \boldsymbol{T}_{\mathrm{A}} \overline{\boldsymbol{\delta}}(t)
$$

So, we have

$$
\frac{\mathrm{d} \overline{\boldsymbol{\delta}}(t)}{\mathrm{d} t}=\boldsymbol{T}_{\mathrm{A}}^{-1} \boldsymbol{A} \mathbf{i} \boldsymbol{T}_{\mathrm{A}} \overline{\boldsymbol{\delta}}(t)=\boldsymbol{J}_{\mathrm{A}} \overline{\boldsymbol{\delta}}(t)
$$

Then, solving Eq. (5.23), we get

$$
\overline{\boldsymbol{\delta}}(t)=\exp \left(\boldsymbol{J}_{\mathrm{A}} t\right) \overline{\boldsymbol{\delta}}(0)
$$

where $\overline{\boldsymbol{\delta}}(0)=\boldsymbol{T}_{\mathrm{A}}^{-1} \hat{\boldsymbol{\delta}}(0)$

So, we can obtain

$\|\overline{\boldsymbol{\delta}}(t)\|=\left\|\exp \left(\boldsymbol{J}_{\mathrm{A}} t\right) \overline{\boldsymbol{\delta}}(0)\right\| \leq\left\|\exp \left(\boldsymbol{J}_{\mathrm{A}} t\right)\right\|\|\overline{\boldsymbol{\delta}}(0)\| \leq\left\|\exp \left(\operatorname{Re} \boldsymbol{J}_{\mathrm{A}} t\right)\right\|\|\overline{\boldsymbol{\delta}}(0)\| \leq \exp \left(-\alpha_{\mathrm{A}} t\right)\|\overline{\boldsymbol{\delta}}(0)\|$

Based on the above analysis, we have 


$$
\begin{aligned}
& \left\|\boldsymbol{x}(t)-\overline{\boldsymbol{x}}_{\mathrm{m} 4}\right\|=\|\hat{\boldsymbol{x}}(t)-\hat{\boldsymbol{\delta}}(t)-\hat{\boldsymbol{x}}(\infty)\| \leq\|\hat{\boldsymbol{x}}(t)-\hat{\boldsymbol{x}}(\infty)\|+\|\hat{\boldsymbol{\delta}}(t)\| \\
& =\left\|\boldsymbol{T}_{\mathrm{m} 4}(\overline{\boldsymbol{x}}(t)-\overline{\boldsymbol{x}}(\infty))\right\|+\left\|\boldsymbol{T}_{\mathrm{A}} \overline{\boldsymbol{\delta}}(t)\right\| \leq\left\|\boldsymbol{T}_{\mathrm{m}} 4\right\|\|\overline{\boldsymbol{x}}(t)-\overline{\boldsymbol{x}}(\infty)\|+\left\|\boldsymbol{T}_{\mathrm{A}}\right\|\|\overline{\boldsymbol{\delta}}(t)\| \\
& \leq\left\|\boldsymbol{T}_{\mathrm{m} 4}\right\| \exp \left(-\alpha_{\mathrm{m} 4} t\right)\|\overline{\boldsymbol{x}}(0)-\overline{\boldsymbol{x}}(\infty)\|+\left\|\boldsymbol{T}_{\mathrm{A}}\right\| \exp \left(-\alpha_{\mathrm{A}} t\right)\|\overline{\boldsymbol{\delta}}(0)\| \\
& =\left\|\boldsymbol{T}_{\mathrm{m} 4}\right\| \exp \left(-\alpha_{\mathrm{m} 4} t\right)\left\|\boldsymbol{T}_{\mathrm{m} 4}{ }^{-1}(\hat{\boldsymbol{x}}(0)-\hat{\boldsymbol{x}}(\infty))\right\|+\left\|\boldsymbol{T}_{\mathrm{A}}\right\| \exp \left(-\alpha_{\mathrm{A}} t\right)\left\|\boldsymbol{T}_{\mathrm{A}}^{-1} \hat{\boldsymbol{\delta}}(0)\right\| \\
& \leq \exp \left(-\alpha_{\mathrm{m} 4} t\right)\left\|\boldsymbol{T}_{\mathrm{m} 4}\right\|\left\|\boldsymbol{T}_{\mathrm{m} 4}{ }^{-1}\right\|\|\hat{\boldsymbol{x}}(0)-\hat{\boldsymbol{x}}(\infty)\|+\exp \left(-\alpha_{\mathrm{A}} t\right)\left\|\boldsymbol{T}_{\mathrm{A}}\right\|\left\|\boldsymbol{T}_{\mathrm{A}}{ }^{-1}\right\|\|\hat{\boldsymbol{\delta}}(0)\| \\
& =\exp \left(-\alpha_{\mathrm{m} 4} t\right)\left\|\boldsymbol{T}_{\mathrm{m} 4}\right\|\left\|\boldsymbol{T}_{\mathrm{m} 4}{ }^{-1}\right\|\left\|\boldsymbol{x}(0)+\hat{\boldsymbol{\delta}}(0)-\overline{\boldsymbol{x}}_{\mathrm{m} 4}\right\|+\exp \left(-\alpha_{\mathrm{A}} t\right)\left\|\boldsymbol{T}_{\mathrm{A}}\right\|\left\|\boldsymbol{T}_{\mathrm{A}}{ }^{-1}\right\|\|\hat{\boldsymbol{\delta}}(0)\| \\
& \leq \exp \left(-\alpha_{\mathrm{m} 4} t\right)\left\|\boldsymbol{T}_{\mathrm{m} 4} 4\right\| \boldsymbol{T}_{\mathrm{m} 4}{ }^{-1}\left\|\left(\|\hat{\boldsymbol{\delta}}(0)\|+\left\|\boldsymbol{x}(0)-\overline{\boldsymbol{x}}_{\mathrm{m} 4}\right\|\right)+\exp \left(-\alpha_{\mathrm{A}} t\right)\right\| \boldsymbol{T}_{\mathrm{A}}\|\| \boldsymbol{T}_{\mathrm{A}}{ }^{-1}\|\| \hat{\boldsymbol{\delta}}(0) \| \\
& =\exp \left(-\alpha_{\mathrm{m} 4} t\right) \operatorname{cond}\left(\boldsymbol{T}_{\mathrm{m} 4}\right)\left(\|\hat{\boldsymbol{\delta}}(0)\|+\left\|\boldsymbol{x}(0)-\overline{\boldsymbol{x}}_{\mathrm{m} 4}\right\|\right)+\exp \left(-\alpha_{\mathrm{A}} t\right) \operatorname{cond}\left(\boldsymbol{T}_{\mathrm{A}}\right)\|\hat{\boldsymbol{\delta}}(0)\|
\end{aligned}
$$

End.

As the checking rate is time-varying parameter, we have

$$
\frac{\mathrm{d} \hat{\boldsymbol{x}}(t)}{\mathrm{d} t}=(\boldsymbol{A}-\boldsymbol{U}(t)) \hat{\boldsymbol{x}}(t)+\boldsymbol{\mu}
$$

Applying $\hat{\boldsymbol{x}}(t)$ to replace $\boldsymbol{x}(t)$ in the control laws (4.4), (4.7) and (4.9), and then we can obtain

$$
\begin{gathered}
\boldsymbol{u}(t)=\left[\begin{array}{l}
u_{1}(t) \\
u_{2}(t)
\end{array}\right]=[\hat{\boldsymbol{X}}(t)]^{-1}\left[\left(\boldsymbol{A}+\boldsymbol{K}_{\mathrm{m} 1)} \hat{\boldsymbol{x}}(t)+\boldsymbol{\mu}-\boldsymbol{K}_{\mathrm{m} 1} \overline{\boldsymbol{x}}_{\mathrm{m} 1}\right]\right. \\
\boldsymbol{u}(t)=\left[\begin{array}{l}
u_{1}(t) \\
u_{2}(t)
\end{array}\right]=\left[\begin{array}{c}
\frac{\mu_{1}+a \bar{x}_{2 \mathrm{~m} 2}}{\bar{x}_{1 \mathrm{~m} 2}}-d_{1} \\
\frac{\left(k_{23}+b\right) \hat{x}_{1}(t)+\left(k_{24}-d_{2}\right) \hat{x}_{2}(t)+\left(\mu_{2}-k_{23} \bar{x}_{1 \mathrm{~m} 2}-k_{24} \bar{x}_{2 \mathrm{~m} 2}\right)}{\hat{x}_{2}(t)}
\end{array}\right] \\
\boldsymbol{u}(t)=\left[\begin{array}{l}
u_{1}(t) \\
u_{2}(t)
\end{array}\right]=\left[\begin{array}{c}
\frac{\left(k_{31}-d_{1}\right) \hat{x}_{1}(t)+\left(k_{32}+a\right) \hat{x}_{2}(t)+\left(\mu_{1}-k_{31} \bar{x}_{\mathrm{m} 3}-k_{32} \bar{x}_{2 \mathrm{~m} 3}\right)}{\hat{x}_{1}(t)} \\
\frac{\mu_{2}+b \bar{x}_{1 \mathrm{~m} 3}}{\bar{x}_{2 \mathrm{~m} 3}}-d_{2}
\end{array}\right]
\end{gathered}
$$

Theorem 4. In the area (1), (2) and (3), under the action of the control laws (5.28), (5.29) and (5.30), the state probability $\boldsymbol{x}(t)$ of the system satisfies, respectively

$$
\begin{aligned}
& \left\|\boldsymbol{x}(t)-\overline{\boldsymbol{x}}_{\mathrm{m} 1}\right\| \leq \exp \left(-\alpha_{\mathrm{m} 1} t\right) \operatorname{cond}\left(\boldsymbol{T}_{\mathrm{m} 1)}\left(\|\hat{\boldsymbol{\delta}}(0)\|+\left\|\boldsymbol{x}(0)-\overline{\boldsymbol{x}}_{\mathrm{m}}\right\|\right)+\exp \left(-\alpha_{\mathrm{M}} t\right) \operatorname{cond}\left(\boldsymbol{T}_{\mathrm{M}}\right)\|\hat{\boldsymbol{\delta}}(0)\|\right. \\
& \left\|\boldsymbol{x}(t)-\overline{\boldsymbol{x}}_{\mathrm{m} 2}\right\| \leq \exp \left(-\alpha_{\mathrm{m} 2} t\right) \operatorname{cond}\left(\boldsymbol{T}_{\mathrm{m} 2}\right)\left(\|\hat{\boldsymbol{\delta}}(0)\|+\left\|\boldsymbol{x}(0)-\overline{\boldsymbol{x}}_{\mathrm{m} 2}\right\|\right)+\exp \left(-\alpha_{\mathrm{N}} t\right) \operatorname{cond}\left(\boldsymbol{T}_{\mathrm{N}}\right)\|\hat{\boldsymbol{\delta}}(0)\| \\
& \left\|\boldsymbol{x}(t)-\overline{\boldsymbol{x}}_{\mathrm{m} 3}\right\| \leq \exp \left(-\alpha_{\mathrm{m} 3} t\right) \operatorname{cond}\left(\boldsymbol{T}_{\mathrm{m} 3}\right)\left(\|\hat{\boldsymbol{\delta}}(0)\|+\left\|\boldsymbol{x}(0)-\overline{\boldsymbol{x}}_{\mathrm{m} 3}\right\|\right)+\exp (-\alpha \mathrm{s} t) \operatorname{cond}\left(\boldsymbol{T}_{\mathrm{s}}\right)\|\hat{\boldsymbol{\delta}}(0)\|
\end{aligned}
$$

where

$$
\begin{aligned}
& \boldsymbol{T}_{\mathrm{m} 1}{ }^{-1} \boldsymbol{A}_{\mathrm{m} 1} \boldsymbol{T}_{\mathrm{m} 1}=\boldsymbol{J}_{\mathrm{m} 1}=\operatorname{diag}\left(\lambda_{1}, \lambda_{2}\right), \quad \alpha_{\mathrm{m} 1}=-\max \left\{\operatorname{Re} \lambda_{i}\left(\boldsymbol{A}_{\mathrm{m} 1}\right)\right\}, \quad i=1,2 \\
& \boldsymbol{T}_{\mathrm{m} 2}{ }^{-1} \boldsymbol{A}_{\mathrm{m} 2} \boldsymbol{T}_{\mathrm{m} 2}=\boldsymbol{J}_{\mathrm{m} 2}=\operatorname{diag}\left(\lambda_{1}, \lambda_{2}\right), \alpha_{\mathrm{m} 2}=-\max \left\{\operatorname{Re} \lambda_{i}\left(\boldsymbol{A}_{\mathrm{m} 2}\right)\right\}, \quad i=1,2
\end{aligned}
$$




$$
\begin{gathered}
\boldsymbol{T}_{\mathrm{m} 3}^{-1} \boldsymbol{A}_{\mathrm{m} 3} \boldsymbol{T}_{\mathrm{m} 3}=\boldsymbol{J}_{\mathrm{m} 3}=\operatorname{diag}\left(\lambda_{1}, \lambda_{2}\right), \alpha_{\mathrm{m} 3}=-\max \left\{\operatorname{Re} \lambda_{i}\left(\boldsymbol{A}_{\mathrm{m} 3}\right)\right\}, \quad i=1,2 \\
\boldsymbol{T}_{\mathrm{M}}{ }^{-1} \boldsymbol{M} \boldsymbol{T}_{\mathrm{M}}=\boldsymbol{J}_{\mathrm{M}}=\operatorname{diag}\left(\lambda_{1}, \lambda_{2}\right), \alpha_{\mathrm{M}}=-\max \left\{\operatorname{Re} \lambda_{i}(\boldsymbol{M})\right\}, \quad i=1,2 \\
\boldsymbol{T}_{\mathrm{N}}^{-1} \boldsymbol{N} \boldsymbol{T}_{\mathrm{N}}=\boldsymbol{J}_{\mathrm{N}}=\operatorname{diag}\left(\lambda_{1}, \lambda_{2}\right), \alpha_{\mathrm{N}}=-\max \left\{\operatorname{Re} \lambda_{i}(\boldsymbol{N})\right\}, \quad i=1,2 \\
\boldsymbol{T}_{\mathrm{S}}^{-1} \boldsymbol{S} \boldsymbol{T}_{\mathrm{S}}=\boldsymbol{J}_{\mathrm{S}}=\operatorname{diag}\left(\lambda_{1}, \lambda_{2}\right), \alpha_{\mathrm{S}}=-\max \left\{\operatorname{Re} \lambda_{i}(\boldsymbol{S})\right\}, \quad i=1,2
\end{gathered}
$$

where $\boldsymbol{M}=\left[\begin{array}{ll}m_{11} & m_{12} \\ m_{21} & m_{22}\end{array}\right], \boldsymbol{N}=\left[\begin{array}{ll}n_{11} & n_{12} \\ n_{21} & n_{22}\end{array}\right]$ and $\boldsymbol{S}=\left[\begin{array}{ll}s_{11} & s_{12} \\ s_{21} & s_{22}\end{array}\right]$ satisfy, respectively

$$
\begin{gathered}
m_{11} \geq a_{11}(t), m_{22} \geq a_{22}(t), m_{12} \geq\left|a_{12}(t)\right|, m_{21} \geq\left|a_{21}(t)\right| . \\
n_{11} \geq b_{11}(t), n_{22} \geq b_{22}(t), n_{12} \geq\left|b_{12}(t)\right|, n_{21} \geq\left|b_{21}(t)\right| . \\
s_{11} \geq c_{11}(t), s_{22} \geq c_{22}(t), s_{12} \geq\left|c_{12}(t)\right|, s_{21} \geq\left|c_{21}(t)\right| .
\end{gathered}
$$

where

$$
\begin{gathered}
{\left[\begin{array}{ll}
a_{11}(t) & a_{12}(t) \\
a_{21}(t) & a_{22}(t)
\end{array}\right]=\left[\begin{array}{cc}
-\left(\lambda_{2}+v_{1}+u_{1}(t)+\mu_{1}\right) & \lambda_{1}-\mu_{1} \\
\lambda_{2}-\mu_{2} & -\left(\lambda_{1}+v_{2}+u_{2}(t)+\mu_{2}\right)
\end{array}\right]=\boldsymbol{A}_{1}(t)} \\
{\left[\begin{array}{ll}
b_{11}(t) & b_{12}(t) \\
b_{21}(t) & b_{22}(t)
\end{array}\right]=\left[\begin{array}{cc}
-\left(\lambda_{2}+v_{1}+u_{1}+\mu_{1}\right) & \lambda_{1}-\mu_{1} \\
\lambda_{2}-\mu_{2} & -\left(\lambda_{1}+v_{2}+u_{2}(t)+\mu_{2}\right)
\end{array}\right]=\boldsymbol{A}_{2}(t)} \\
{\left[\begin{array}{ll}
c_{11}(t) & c_{12}(t) \\
c_{21}(t) & c_{22}(t)
\end{array}\right]=\left[\begin{array}{cc}
-\left(\lambda_{2}+v_{1}+u_{1}(t)+\mu_{1}\right) & \lambda_{1}-\mu_{1} \\
\lambda_{2}-\mu_{2} & -\left(\lambda_{1}+v_{2}+u_{2}+\mu_{2}\right)
\end{array}\right]=\boldsymbol{A}_{3}(t)}
\end{gathered}
$$

Proof. Under the action of control law (5.28), Eq. (5.27) will become the following form.

$$
\frac{\mathrm{d} \hat{\boldsymbol{x}}(t)}{\mathrm{d} t}=\boldsymbol{A}_{\mathrm{m} 1} \hat{\boldsymbol{x}}(t)+\boldsymbol{\mu}_{\mathrm{m} 1}
$$

Then, we Let

$$
\hat{\boldsymbol{x}}(t)=\boldsymbol{T}_{\mathrm{m} 1} \overline{\boldsymbol{x}}(t)
$$

where

$$
\boldsymbol{T}_{\mathrm{m} 1}^{-1} \boldsymbol{A}_{\mathrm{m} 1} \boldsymbol{T}_{\mathrm{m} 1}=\boldsymbol{J}_{\mathrm{m} 1}=\operatorname{diag}\left(\lambda_{1}, \lambda_{2}\right)
$$

And then, we let

$$
\alpha \mathrm{ml}=-\max \left\{\operatorname{Re} \lambda_{i}\left(\boldsymbol{A}_{\mathrm{m} 1}\right)\right\}, \quad i=1,2
$$

According Eq. (5.34) and Eq. (5.35), we can obtain

$$
\frac{\mathrm{d} \boldsymbol{T}_{\mathrm{m} 1} \overline{\boldsymbol{x}}(t)}{\mathrm{d} t}=\boldsymbol{A}_{\mathrm{m} 1} \boldsymbol{T}_{\mathrm{m} 1} \overline{\boldsymbol{x}}(t)+\boldsymbol{\mu}_{\mathrm{m} 1}
$$

So, we have 


$$
\frac{\mathrm{d} \overline{\boldsymbol{x}}(t)}{\mathrm{d} t}=\boldsymbol{T}_{\mathrm{m} 1}{ }^{-1} \boldsymbol{A}_{\mathrm{m} 1} \boldsymbol{T}_{\mathrm{m} 1} \overline{\boldsymbol{x}}(t)+\boldsymbol{T}_{\mathrm{m} 1}{ }^{-1} \boldsymbol{\mu}_{\mathrm{m} 1}=\boldsymbol{J}_{\mathrm{m} 1} \overline{\boldsymbol{x}}(t)+\boldsymbol{T}_{\mathrm{m} 1}{ }^{-1} \boldsymbol{\mu}_{\mathrm{m} 1}
$$

Then, solving Eq. (5.37), we get

$$
\begin{aligned}
\overline{\boldsymbol{x}}(t) & =\exp \left(\boldsymbol{J}_{\mathrm{m} 1} t\right) \overline{\boldsymbol{x}}(0)+\int_{0}^{t} \exp \left(\boldsymbol{J}_{\mathrm{m} 1}(t-\tau)\right) \mathrm{d} \tau \cdot \boldsymbol{T}_{\mathrm{m} 1}{ }^{-1} \boldsymbol{\mu}_{\mathrm{m} 1} . \\
& =\exp \left(\boldsymbol{J}_{\mathrm{m} 1} t\right)(\overline{\boldsymbol{x}}(0)-\overline{\boldsymbol{x}}(\infty))+\overline{\boldsymbol{x}}(\infty)
\end{aligned}
$$

where $\overline{\boldsymbol{x}}(0)=\boldsymbol{T}_{\mathrm{m} 1}{ }^{-1} \hat{\boldsymbol{x}}(0)$ and $\overline{\boldsymbol{x}}(\infty)=\boldsymbol{T}_{\mathrm{m} 1}{ }^{-1} \hat{\boldsymbol{x}}(\infty)=-\boldsymbol{J}_{\mathrm{m} 1}{ }^{-1} \boldsymbol{T}_{\mathrm{m} 1}{ }^{-1} \boldsymbol{\mu}_{\mathrm{m} 1}=-\boldsymbol{T}_{\mathrm{m} 1}{ }^{-1} \boldsymbol{A}_{\mathrm{m} 1}{ }^{-1} \boldsymbol{\mu}_{\mathrm{m} 1}=\boldsymbol{T}_{\mathrm{m} 1}{ }^{-1} \overline{\boldsymbol{x}}_{\mathrm{m} 1}$. So, we can obtain

$$
\overline{\boldsymbol{x}}(t)-\overline{\boldsymbol{x}}(\infty)=\exp \left(\boldsymbol{J}_{\mathrm{m} 1} t\right)(\overline{\boldsymbol{x}}(0)-\overline{\boldsymbol{x}}(\infty))
$$

Based on the above analysis, we have

$$
\begin{aligned}
\|\overline{\boldsymbol{x}}(t)-\overline{\boldsymbol{x}}(\infty)\| & =\left\|\exp \left(\boldsymbol{J}_{\mathrm{m} 1} t\right)(\overline{\boldsymbol{x}}(0)-\overline{\boldsymbol{x}}(\infty))\right\| \leq\left\|\exp \left(\boldsymbol{J}_{\mathrm{m} \mathrm{l}} t\right)\right\|\|\overline{\boldsymbol{x}}(0)-\overline{\boldsymbol{x}}(\infty)\| \\
& \leq\left\|\exp \left(\operatorname{Re} \boldsymbol{J}_{\mathrm{m} 1} t\right)\right\|\|\overline{\boldsymbol{x}}(0)-\overline{\boldsymbol{x}}(\infty)\| \leq \exp \left(-\alpha_{\mathrm{m} 1} t\right)\|\overline{\boldsymbol{x}}(0)-\overline{\boldsymbol{x}}(\infty)\|
\end{aligned}
$$

So, we can obtain

$$
\begin{aligned}
& \|\hat{\boldsymbol{x}}(t)-\hat{\boldsymbol{x}}(\infty)\|=\left\|\boldsymbol{T}_{\mathrm{m}}(\overline{\boldsymbol{x}}(t)-\overline{\boldsymbol{x}}(\infty))\right\| \leq\left\|\boldsymbol{T}_{\mathrm{m}} \mid\right\| \overline{\boldsymbol{x}}(t)-\overline{\boldsymbol{x}}(\infty)\|\leq\| \boldsymbol{T}_{\mathrm{m}}\left\|\exp \left(-\alpha_{\mathrm{m}} t\right)\right\| \overline{\boldsymbol{x}}(0)-\overline{\boldsymbol{x}}(\infty) \| \\
& =\left\|\boldsymbol{T}_{\mathrm{m}}\right\| \exp \left(-\alpha_{\mathrm{m} \mathrm{m}} t\right)\left\|\boldsymbol{T}_{\mathrm{m} 1}{ }^{-1}(\hat{\boldsymbol{x}}(0)-\hat{\boldsymbol{x}}(\infty))\right\| \leq \exp \left(-\alpha_{\mathrm{m} !} t\right)\left\|\boldsymbol{T}_{\mathrm{m}}\right\|\left\|\boldsymbol{T}_{\mathrm{m} 1}{ }^{-1}\right\|\|\hat{\boldsymbol{x}}(0)-\hat{\boldsymbol{x}}(\infty)\|
\end{aligned}
$$

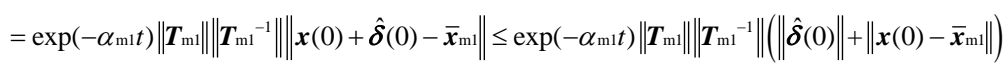

Then, we let

$$
\frac{\mathrm{d} \hat{\boldsymbol{\delta}}(t)}{\mathrm{d} t}=(\boldsymbol{A}-\boldsymbol{U}(t)) \hat{\boldsymbol{\delta}}(t)=\boldsymbol{A}_{1}(t) \hat{\boldsymbol{\delta}}(t)
$$

where

$$
\boldsymbol{A}_{1}(t)=\left[\begin{array}{ll}
a_{11}(t) & a_{12}(t) \\
a_{21}(t) & a_{22}(t)
\end{array}\right]=\left[\begin{array}{cc}
-\left(\lambda_{2}+v_{1}+u_{1}(t)+\mu_{1}\right) & \lambda_{1}-\mu_{1} \\
\lambda_{2}-\mu_{2} & -\left(\lambda_{1}+v_{2}+u_{2}(t)+\mu_{2}\right)
\end{array}\right]
$$

And then, we define

$$
\boldsymbol{M}=\left[\begin{array}{ll}
m_{11} & m_{12} \\
m_{21} & m_{22}
\end{array}\right], \quad \boldsymbol{D}_{\mathrm{M}}=\left[\begin{array}{ll}
m_{11} & \\
& m_{22}
\end{array}\right], \boldsymbol{D}_{\mathrm{A}}(t)=\left[\begin{array}{ll}
a_{11}(t) & \\
& a_{22}(t)
\end{array}\right]
$$

where $m_{11} \geq a_{11}(t), m_{22} \geq a_{22}(t), m_{12} \geq\left|a_{12}(t)\right|, m_{21} \geq\left|a_{21}(t)\right|$.

We define a system expressed by the following form.

$$
\frac{\mathrm{d} \delta(t)}{\mathrm{d} t}=\boldsymbol{M} \boldsymbol{\delta}^{(}(t)
$$

where $\delta^{\prime}(0)=|\hat{\boldsymbol{\delta}}(0)|$.

Then, we let $\boldsymbol{A}_{1}(t)=\boldsymbol{D}_{\mathrm{A}}(t)+\boldsymbol{A}_{1}(t)-\boldsymbol{D}_{\mathrm{A}}(t)$. So, we can obtain

$$
\frac{\mathrm{d} \hat{\boldsymbol{\delta}}(t)}{\mathrm{d} t}=\boldsymbol{A}_{1}(t) \hat{\boldsymbol{\delta}}(t)=\left(\boldsymbol{D}_{\mathrm{A}}(t)+\boldsymbol{A}_{1}(t)-\boldsymbol{D}_{\mathrm{A}}(t)\right) \hat{\boldsymbol{\delta}}(t)
$$

And then, solving Eq. (5.44). Thus, we have 


$$
\hat{\boldsymbol{\delta}}(t)=\exp \left(\int_{0}^{t} \boldsymbol{D}_{\mathrm{A}}(\tau) \mathrm{d} \tau\right) \hat{\boldsymbol{\delta}}(0)+\int_{0}^{t} \exp \left(\int_{\tau}^{t} \boldsymbol{D}_{\mathrm{A}}(\eta) \mathrm{d} \eta\right)\left(\boldsymbol{A}_{1}(\tau)-\boldsymbol{D}_{\mathrm{A}}(\tau)\right) \hat{\boldsymbol{\delta}}(\tau) \mathrm{d} \tau
$$

It is quite obvious that $\boldsymbol{D}_{\AA}(t) \leq \boldsymbol{D}_{\mathrm{M}}$ and $\left|\boldsymbol{A}_{1}(t)-\boldsymbol{D}_{\AA}(t)\right| \leq \boldsymbol{M}-\boldsymbol{D}_{\mathrm{M}}$. So, we get

$$
\begin{aligned}
|\hat{\boldsymbol{\delta}}(t)| & \leq \exp \left(\int_{0}^{t} \boldsymbol{D}_{\mathrm{A}}(\tau) \mathrm{d} \tau\right)|\hat{\boldsymbol{\delta}}(0)|+\int_{0}^{t} \exp \left(\int_{\tau}^{t} \boldsymbol{D}_{\mathrm{A}}(\eta) \mathrm{d} \eta\right)\left|\boldsymbol{A}_{1}(\tau)-\boldsymbol{D}_{\mathrm{A}}(\tau)\right| \hat{\boldsymbol{\delta}}(\tau) \mid \mathrm{d} \tau \\
& \leq \exp \left(\int_{0}^{t} \boldsymbol{D}_{\mathrm{M}} \mathrm{d} \tau\right)|\hat{\boldsymbol{\delta}}(0)|+\int_{0}^{t} \exp \left(\int_{\tau}^{t} \boldsymbol{D}_{\mathrm{M}} \mathrm{d} \eta\right)\left(\boldsymbol{M}-\boldsymbol{D}_{\mathrm{M}}\right)|\hat{\boldsymbol{\delta}}(\tau)| \mathrm{d} \tau \\
& =\exp \left(\boldsymbol{D}_{\mathrm{M}} t\right)|\hat{\boldsymbol{\delta}}(0)|+\int_{0}^{t} \exp \left(\boldsymbol{D}_{\mathrm{M}}(t-\tau)\right)\left(\boldsymbol{M}-\boldsymbol{D}_{\mathrm{M}}\right)|\hat{\boldsymbol{\delta}}(\tau)| \mathrm{d} \tau
\end{aligned}
$$

Then, we Let

$$
\grave{\delta}^{\prime}(t)=\exp \left(\boldsymbol{D}_{\mathrm{M}} t\right)|\hat{\boldsymbol{\delta}}(0)|+\int_{0}^{t} \exp \left(\boldsymbol{D}_{\mathrm{M}}(t-\tau)\right)\left(\boldsymbol{M}-\boldsymbol{D}_{\mathrm{M}}\right)|\hat{\boldsymbol{\delta}}(\tau)| \mathrm{d} \tau
$$

According Eq. (5.46) and Eq. (5.47), we have $|\hat{\boldsymbol{\delta}}(t)| \leq \hat{\boldsymbol{\delta}}(t)$.

So, from Eq. (5.47) we can obtain

$$
\begin{aligned}
\frac{\mathrm{d} \hat{\boldsymbol{\delta}}(t)}{\mathrm{d} t} & =\boldsymbol{D}_{\mathrm{M}} \exp \left(\boldsymbol{D}_{\mathrm{M}} t\right)|\hat{\boldsymbol{\delta}}(0)|+\boldsymbol{D}_{\mathrm{M}} \int_{0}^{t} \exp \left(\boldsymbol{D}_{\mathrm{M}}(t-\tau)\right)\left(\boldsymbol{M}-\boldsymbol{D}_{\mathrm{M}}\right)|\hat{\boldsymbol{\delta}}(\tau)| \mathrm{d} \tau+\left(\boldsymbol{M}-\boldsymbol{D}_{\mathrm{M}}\right)|\hat{\boldsymbol{\delta}}(t)| \\
& =\boldsymbol{D}_{\mathrm{M}} \hat{\boldsymbol{\delta}}(t)+\left(\boldsymbol{M}-\boldsymbol{D}_{\mathrm{M}}\right)|\hat{\boldsymbol{\delta}}(t)| \leq \boldsymbol{D}_{\mathrm{M}} \boldsymbol{\delta}(t)+\left(\boldsymbol{M}-\boldsymbol{D}_{\mathrm{M}}\right) \boldsymbol{\delta}(t)=\boldsymbol{M} \boldsymbol{\delta}(t)
\end{aligned}
$$

According to vector comparison principle [16], we have

$$
|\hat{\boldsymbol{\delta}}(t)| \leq \hat{\boldsymbol{\delta}}(t) \leq \delta(t)
$$

Then, we let

$$
\delta(t)=\boldsymbol{T}_{\mathrm{M}} \stackrel{\vee}{\boldsymbol{\delta}}(t)
$$

where

$$
\boldsymbol{T}_{\mathrm{M}}^{-1} \boldsymbol{M} \boldsymbol{T}_{\mathrm{M}}=\boldsymbol{J}_{\mathrm{M}}=\operatorname{diag}\left(\lambda_{1}, \lambda_{2}\right)
$$

And then, we let

$$
\alpha_{\mathrm{M}}=-\max \left\{\operatorname{Re} \lambda_{i}(\boldsymbol{M})\right\}, \quad i=1,2
$$

According Eq. (5.43) and Eq. (5.50), we can obtain

$$
\frac{\mathrm{d} \boldsymbol{T}_{\mathrm{M}} \stackrel{\vee}{\boldsymbol{\delta}}(t)}{\mathrm{d} t}=\boldsymbol{M} \boldsymbol{T}_{\mathrm{M}} \stackrel{\mathrm{V}}{\mathrm{V}}(t)
$$

So, we have

$$
\frac{\mathrm{d} \dot{\boldsymbol{\delta}}(t)}{\mathrm{d} t}=\boldsymbol{T}_{\mathrm{M}}^{-1} \boldsymbol{M} \boldsymbol{T}_{\mathrm{M}} \stackrel{\mathrm{V}}{\mathrm{V}}(t)=\boldsymbol{J}_{\mathrm{M}} \stackrel{\mathrm{V}}{\boldsymbol{\delta}}(t)
$$

Then, solving Eq. (5.52), we get

$$
\stackrel{\vee}{\boldsymbol{\delta}}(t)=\exp \left(\boldsymbol{J}_{\mathrm{M}} t\right) \stackrel{\mathrm{v}}{\boldsymbol{\delta}}(0)
$$

where $\stackrel{\vee}{\delta}(0)=\boldsymbol{T}_{\mathrm{M}}^{-1} \delta(0)$. 
So, we have

$\|\overrightarrow{\boldsymbol{\delta}}(t)\|=\left\|\exp \left(\boldsymbol{J}_{\mathrm{M}} t\right) \overrightarrow{\boldsymbol{\delta}}(0)\right\| \leq\left\|\exp \left(\boldsymbol{J}_{\mathrm{M}} t\right)\right\|\|\overrightarrow{\boldsymbol{\delta}}(0)\| \leq\left\|\exp \left(\operatorname{Re} \boldsymbol{J}_{\mathrm{M}} t\right)\right\|\|\overrightarrow{\boldsymbol{\delta}}(0)\| \leq \exp \left(-\alpha_{\mathrm{M}} t\right)\|\overrightarrow{\boldsymbol{\delta}}(0)\|$

Thus, we can obtain

$$
\begin{aligned}
\|\hat{\boldsymbol{\delta}}(t)\| & \leq\|\boldsymbol{\delta}(t)\|=\left\|\boldsymbol{T}_{\mathrm{M}} \boldsymbol{\mathrm { \delta }}(t)\right\| \leq\left\|\boldsymbol{T}_{\mathrm{M}}\right\|\|\boldsymbol{\delta}(t)\| \leq\left\|\boldsymbol{T}_{\mathrm{M}}\right\| \exp \left(-\alpha_{\mathrm{M}} t\right)\|\boldsymbol{\delta}(0)\|=\left\|\boldsymbol{T}_{\mathrm{M}}\right\| \exp \left(-\alpha_{\mathrm{M}} t\right)\left\|\boldsymbol{T}_{\mathrm{M}}{ }^{-1} \boldsymbol{\delta}(0)\right\| \\
& \leq \exp \left(-\alpha_{\mathrm{M}} t\right)\left\|\boldsymbol{T}_{\mathrm{M}}\right\|\left\|\boldsymbol{T}_{\mathrm{M}}{ }^{-1}\right\|\|\boldsymbol{\delta}(0)\|=\exp \left(-\alpha_{\mathrm{M}} t\right)\left\|\boldsymbol{T}_{\mathrm{M}}\right\|\left\|\boldsymbol{T}_{\mathrm{M}}{ }^{-1}\right\|\left\|\hat{\boldsymbol{\delta}}_{(0)}\right\|
\end{aligned}
$$

Based on the above analysis, we have

$$
\begin{aligned}
\left\|\boldsymbol{x}(t)-\overline{\boldsymbol{x}}_{\mathrm{m} 1}\right\| & =\|\hat{\boldsymbol{x}}(t)-\hat{\boldsymbol{\delta}}(t)-\hat{\boldsymbol{x}}(\infty)\| \leq\|\hat{\boldsymbol{x}}(t)-\hat{\boldsymbol{x}}(\infty)\|+\|\hat{\boldsymbol{\delta}}(t)\| \\
& \leq \exp \left(-\alpha_{\mathrm{m}} t\right)\left\|\boldsymbol{T}_{\mathrm{m} 1}\right\|\left\|\boldsymbol{T}_{\mathrm{m} 1}{ }^{-1}\right\|\left(\|\hat{\boldsymbol{\delta}}(0)\|+\left\|\boldsymbol{x}(0)-\overline{\boldsymbol{x}}_{\mathrm{m} 1}\right\|\right)+\exp \left(-\alpha_{\mathrm{M}} t\right)\left\|\boldsymbol{T}_{\mathrm{M}}\right\|\left\|\boldsymbol{T}_{\mathrm{M}}{ }^{-1}\right\|\|\hat{\boldsymbol{\delta}}(0)\| \\
& =\exp \left(-\alpha_{\mathrm{m} 1} t\right) \operatorname{cond}\left(\boldsymbol{T}_{\mathrm{m} 1)}\left(\|\hat{\boldsymbol{\delta}}(0)\|+\left\|\boldsymbol{x}(0)-\overline{\boldsymbol{x}}_{\mathrm{m} 1}\right\|\right)+\exp \left(-\alpha_{\mathrm{M}} t\right) \operatorname{cond}\left(\boldsymbol{T}_{\mathrm{M}}\right)\|\hat{\boldsymbol{\delta}}(0)\|\right.
\end{aligned}
$$

The proof processes of Eq. (5.32) and Eq. (5.33) are similar to the proof process of Eq. (5.31). Thus, here the processes are ignored.

End.

According to literature [17], we can obtain

$$
\operatorname{cond}(\boldsymbol{A})=\|\boldsymbol{A}\|_{2}\left\|\boldsymbol{A}^{-1}\right\|_{2}=1
$$

where the matrix $\boldsymbol{A}$ is a orthogonal matrix and $\|\boldsymbol{A}\|_{2}$ denotes the spectrum norm of the matrix $\boldsymbol{A}$.

Thus, if $\boldsymbol{T}_{\mathrm{m} 4}, \boldsymbol{T}_{\mathrm{A}}, \boldsymbol{T}_{\mathrm{m} 1}, \boldsymbol{T}_{\mathrm{M}}, \boldsymbol{T}_{\mathrm{m} 2}, \boldsymbol{T}_{\mathrm{N}}, \boldsymbol{T}_{\mathrm{m} 3}$ and $\boldsymbol{T}_{\mathrm{s}}$ that are described in Theorem 3 and Theorem 4 are orthogonal matrixes, then we can obtain

$$
\begin{aligned}
& \left\|\boldsymbol{x}(t)-\overline{\boldsymbol{x}}_{\mathrm{m} 4}\right\| \leq \exp \left(-\alpha_{\mathrm{m} 4} t\right)\left(\|\hat{\boldsymbol{\delta}}(0)\|+\left\|\boldsymbol{x}(0)-\overline{\boldsymbol{x}}_{\mathrm{m} 4}\right\|\right)+\exp \left(-\alpha_{\mathrm{A}} t\right)\|\hat{\boldsymbol{\delta}}(0)\| \\
& \left\|\boldsymbol{x}(t)-\overline{\boldsymbol{x}}_{\mathrm{m} 1}\right\| \leq \exp \left(-\alpha_{\mathrm{m} 1} t\right)\left(\|\hat{\boldsymbol{\delta}}(0)\|+\left\|\boldsymbol{x}(0)-\overline{\boldsymbol{x}}_{\mathrm{m} 1}\right\|\right)+\exp \left(-\alpha_{\mathrm{M}} t\right)\|\hat{\boldsymbol{\delta}}(0)\| \\
& \left\|\boldsymbol{x}(t)-\overline{\boldsymbol{x}}_{\mathrm{m} 2}\right\| \leq \exp \left(-\alpha_{\mathrm{m} 2} t\right)\left(\|\hat{\boldsymbol{\delta}}(0)\|+\left\|\boldsymbol{x}(0)-\overline{\boldsymbol{x}}_{\mathrm{m} 2}\right\|\right)+\exp \left(-\alpha_{\mathrm{N}} t\right)\|\hat{\boldsymbol{\delta}}(0)\| \\
& \left\|\boldsymbol{x}(t)-\overline{\boldsymbol{x}}_{\mathrm{m} 3}\right\| \leq \exp \left(-\alpha_{\mathrm{m} 3} t\right)\left(\|\hat{\boldsymbol{\delta}}(0)\|+\left\|\boldsymbol{x}(0)-\overline{\boldsymbol{x}}_{\mathrm{m} \mathrm{3}}\right\|\right)+\exp \left(-\alpha_{\mathrm{s}} t\right)\|\hat{\boldsymbol{\delta}}(0)\|
\end{aligned}
$$

where $\alpha_{\mathrm{m} 4}, \alpha_{\mathrm{A}}, \alpha_{\mathrm{m} 1}, \alpha_{\mathrm{M}}, \alpha_{\mathrm{m} 2}, \alpha_{\mathrm{N}}, \alpha_{\mathrm{m} 3}$ and $\alpha_{\mathrm{s}}$ satisfy the conditions as showed in Theorem 3 and Theorem 4.

Theorem 5. In the area (1), (2) and (3), under the action of the control laws (5.28), (5.29) and (5.30), the state probability $\boldsymbol{x}(t)$ of the system satisfies, respectively

$$
\left\|\boldsymbol{x}(t)-\overline{\boldsymbol{x}}_{\mathrm{m} \mathrm{l}}\right\| \leq\left\|\boldsymbol{x}(0)-\overline{\boldsymbol{x}}_{\mathrm{m} 1}\right\|\left\|\exp \left(-\boldsymbol{K}_{\mathrm{m} 1} t\right)\right\|+\sigma\|\hat{\boldsymbol{\delta}}(0)\|\left\|\left(\exp \left(-\boldsymbol{K}_{\mathrm{m} \mathrm{l} t}\right)-\exp (-\gamma t)\right)\left(\boldsymbol{\gamma}-\boldsymbol{K}_{\mathrm{m} 1}\right)^{-1}\right\|
$$




$$
\begin{aligned}
& \left\|\boldsymbol{x}(t)-\overline{\boldsymbol{x}}_{\mathrm{m} 2}\right\| \leq\left\|\boldsymbol{x}(0)-\overline{\boldsymbol{x}}_{\mathrm{m} 2}\right\|\left\|\exp \left(-\boldsymbol{K}_{\mathrm{m} 2} t\right)\right\|+\omega\|\hat{\boldsymbol{\delta}}(0)\|\left\|\left(\exp \left(-\boldsymbol{K}_{\mathrm{m} 2} t\right)-\exp (-\boldsymbol{\eta} t)\right)\left(\boldsymbol{\eta}-\boldsymbol{K}_{\mathrm{m} 2}\right)^{-1}\right\| \\
& \left\|\boldsymbol{x}(t)-\overline{\boldsymbol{x}}_{\mathrm{m} 3}\right\| \leq\left\|\boldsymbol{x}(0)-\overline{\boldsymbol{x}}_{\mathrm{m} 3}\right\|\left\|\exp \left(-\boldsymbol{K}_{\mathrm{m} 3} t\right)\right\|+\varepsilon\|\hat{\boldsymbol{\delta}}(0)\|\left\|\left(\exp \left(-\boldsymbol{K}_{\mathrm{m} 3} t\right)-\exp (-\boldsymbol{\psi} t)\right)\left(\boldsymbol{\psi}-\boldsymbol{K}_{\mathrm{m} 3}\right)^{-1}\right\|
\end{aligned}
$$

where

$$
\begin{aligned}
& \boldsymbol{\gamma}=-\boldsymbol{M}, \sigma=\left\|\boldsymbol{\gamma}-\boldsymbol{K}_{\mathrm{m} 1}\right\| \|_{\max }=\max \left\{\left\|\boldsymbol{\gamma}-\boldsymbol{K}_{\mathrm{m}}\right\|\right\} \\
& \boldsymbol{\eta}=-\boldsymbol{N}, \omega=\left\|\boldsymbol{\eta}-\boldsymbol{K}_{\mathrm{m} 2}\right\|_{\max }=\max \left\{\left\|\boldsymbol{\eta}-\boldsymbol{K}_{\mathrm{m} 2}\right\|\right\} \\
& \boldsymbol{\psi}=-\boldsymbol{S}, \varepsilon=\left\|\boldsymbol{\psi}-\boldsymbol{K}_{\mathrm{m}}\right\|_{\max }=\max \left\{\left\|\boldsymbol{\eta}-\boldsymbol{K}_{\mathrm{m}}\right\|\right\}
\end{aligned}
$$

After maximum time $t_{\mathrm{m} 1}, t_{\mathrm{m} 2}$ and $t_{\mathrm{m} 3}$, the second item in Eq. (5.62), Eq. (5.63) and Eq. (5.64) decreases rapidly, respectively, where $t_{\mathrm{m} 1}, t_{\mathrm{m} 2}$ and $t_{\mathrm{m} 3}$ satisfy, respectively

$$
\begin{aligned}
& t_{\mathrm{m} 1}=\max \left\{\boldsymbol{T}_{\mathrm{m} 1}\right\}=\max \left\{\left[\begin{array}{ll}
t_{11} & t_{12} \\
t_{13} & t_{14}
\end{array}\right]\right\} \approx m_{11} \Delta t \\
& t_{\mathrm{m} 2}=\max \left\{\boldsymbol{T}_{\mathrm{m} 2}\right\}=\max \left\{\left[\begin{array}{ll}
t_{21} & t_{22} \\
t_{23} & t_{24}
\end{array}\right]\right\} \approx m_{21} \Delta t \\
& t_{\mathrm{m} 3}=\max \left\{\boldsymbol{T}_{\mathrm{m} 3}\right\}=\max \left\{\left[\begin{array}{ll}
t_{31} & t_{32} \\
t_{33} & t_{34}
\end{array}\right]\right\} \approx m_{31} \Delta t
\end{aligned}
$$

where $\Delta t$ is a small but infinite time interval for an effective implementation, $m_{11}, m_{21}$ and $m_{31}$ are a suitable integer, respectively. where $\boldsymbol{T}_{\mathrm{m} 1}=\left[\begin{array}{ll}t_{11} & t_{12} \\ t_{13} & t_{14}\end{array}\right], \boldsymbol{T}_{\mathrm{m} 2}=\left[\begin{array}{ll}t_{21} & t_{22} \\ t_{23} & t_{24}\end{array}\right]$ and $\boldsymbol{T}_{\mathrm{m} 3}=\left[\begin{array}{ll}t_{31} & t_{32} \\ t_{33} & t_{34}\end{array}\right]$ satisfy the following equations, respectively.

$$
\begin{aligned}
& \gamma_{11} r_{1}\left(t_{11}\right)+\gamma_{2} r_{3}\left(t_{11}\right)-k_{11} f_{11}\left(t_{11}\right)-k_{12} f_{13}\left(t_{11}\right)=0 \\
& \gamma_{1} r_{2}\left(t_{12}\right)+\gamma_{2} r_{4}\left(t_{12}\right)-k_{11} f_{12}\left(t_{12}\right)-k_{12} f_{14}\left(t_{12}\right)=0 \\
& \gamma_{3} r_{1}\left(t_{13}\right)+\gamma_{4} r_{3}\left(t_{13}\right)-k_{13} f_{11}\left(t_{13}\right)-k_{14} f_{13}\left(t_{13}\right)=0 \\
& \gamma_{3} r_{2}\left(t_{11}\right)+\gamma_{4} r_{4}\left(t_{14}\right)-k_{13} f_{12}\left(t_{14}\right)-k_{14} f_{14}\left(t_{14}\right)=0 \\
& \eta_{1} l_{1}\left(t_{21}\right)+\eta_{2} l_{3}\left(t_{21}\right)-k_{21} f_{21}\left(t_{21}\right)-k_{22} f_{23}\left(t_{21}\right)=0 \\
& \eta_{1} l_{2}\left(t_{22}\right)+\eta_{2} l_{4}\left(t_{22}\right)-k_{21} f_{22}\left(t_{22}\right)-k_{22} f_{24}\left(t_{22}\right)=0 \\
& \eta_{3} l_{1}\left(t_{23}\right)+\eta_{4} l_{3}\left(t_{23}\right)-k_{23} f_{21}\left(t_{23}\right)-k_{24} f_{23}\left(t_{23}\right)=0 \\
& \eta_{3} l_{2}\left(t_{24}\right)+\eta_{4} l_{4}\left(t_{24}\right)-k_{23} f_{22}\left(t_{24}\right)-k_{24} f_{24}\left(t_{24}\right)=0
\end{aligned}
$$




$$
\begin{aligned}
& \psi_{1} \varphi_{1}\left(t_{31}\right)+\psi_{2} \varphi_{3}\left(t_{31}\right)-k_{31} f_{31}\left(t_{31}\right)-k_{32} f_{33}\left(t_{31}\right)=0 \\
& \psi_{1} \varphi_{2}\left(t_{32}\right)+\psi_{2} \varphi_{4}\left(t_{32}\right)-k_{31} f_{32}\left(t_{32}\right)-k_{32} f_{34}\left(t_{32}\right)=0 \\
& \psi_{3} \varphi_{1}\left(t_{33}\right)+\psi_{4} \varphi_{3}\left(t_{33}\right)-k_{33} f_{31}\left(t_{33}\right)-k_{34} f_{33}\left(t_{33}\right)=0 \\
& \psi_{3} \varphi_{2}\left(t_{34}\right)+\psi_{4} \varphi_{4}\left(t_{34}\right)-k_{33} f_{32}\left(t_{34}\right)-k_{34} f_{34}\left(t_{34}\right)=0
\end{aligned}
$$

where

$$
\begin{aligned}
& {\left[\begin{array}{ll}
\gamma_{1} & \gamma_{2} \\
\gamma_{3} & \gamma_{4}
\end{array}\right]=\boldsymbol{\gamma},\left[\begin{array}{ll}
r_{1}(t) & r_{2}(t) \\
r_{3}(t) & r_{4}(t)
\end{array}\right]=\exp (-\gamma t)=L^{-1}\left((s \boldsymbol{I}+\boldsymbol{\gamma})^{-1}\right)} \\
& {\left[\begin{array}{ll}
\eta_{1} & \eta_{2} \\
\eta_{3} & \eta_{4}
\end{array}\right]=\boldsymbol{\eta},\left[\begin{array}{ll}
l_{1}(t) & l_{2}(t) \\
l_{3}(t) & l_{4}(t)
\end{array}\right]=\exp (-\boldsymbol{\eta} t)=L^{-1}\left((s \boldsymbol{I}+\boldsymbol{\eta})^{-1}\right)} \\
& {\left[\begin{array}{ll}
\psi_{1} & \psi_{2} \\
\psi_{3} & \psi_{4}
\end{array}\right]=\boldsymbol{\psi},\left[\begin{array}{ll}
\varphi_{1}(t) & \varphi_{2}(t) \\
\varphi_{3}(t) & \varphi_{4}(t)
\end{array}\right]=\exp (-\boldsymbol{\psi} t)=L^{-1}\left((s \boldsymbol{I}+\boldsymbol{\psi})^{-1}\right)}
\end{aligned}
$$

Proof. From Eq. (5.42) we obtain

$$
\hat{\boldsymbol{\delta}}(t)=\exp \left(\int_{0}^{t} \boldsymbol{A}_{1}(\tau) \mathrm{d} \tau\right) \hat{\boldsymbol{\delta}}(0)
$$

According to Eq.(5.43) and Eq. (5.49), we have

$$
\hat{\boldsymbol{\delta}}(t) \leq \boldsymbol{\delta}(t)=\exp (\boldsymbol{M} t)|\hat{\boldsymbol{\delta}}(0)|=\exp (-\gamma t)|\hat{\boldsymbol{\delta}}(0)|
$$

where $\gamma=-\boldsymbol{M}$.

Under the action of control law (5.28), Eq. (5.27) will become the following form.

$$
\frac{\mathrm{d} \hat{\boldsymbol{x}}(t)}{\mathrm{d} t}=-\boldsymbol{K}_{\mathrm{m} 1} \hat{\boldsymbol{x}}(t)+\boldsymbol{K}_{\mathrm{m} 1} \overline{\boldsymbol{x}}_{\mathrm{m} 1}
$$

Then, we can obtain

$$
\hat{\boldsymbol{x}}(t)-\overline{\boldsymbol{x}}_{\mathrm{m} 1}=\exp \left(-\boldsymbol{K}_{\mathrm{m} 1} t\right)\left(\hat{\boldsymbol{x}}(0)-\overline{\boldsymbol{x}}_{\mathrm{m} 1}\right)
$$

Based on the above analysis, we have

$$
\begin{aligned}
& \left\|\boldsymbol{x}(t)-\overline{\boldsymbol{x}}_{\mathrm{m} 1}\right\|=\left\|\hat{\boldsymbol{x}}(t)-\overline{\boldsymbol{x}}_{\mathrm{m} 1}-\hat{\boldsymbol{\delta}}(t)\right\|=\left\|\exp \left(-\boldsymbol{K}_{\mathrm{m} 1} t\right)\left(\hat{\boldsymbol{x}}(0)-\overline{\boldsymbol{x}}_{\mathrm{m} 1}\right)-\hat{\boldsymbol{\delta}}(t)\right\| \\
& =\left\|\exp \left(-\boldsymbol{K}_{\mathrm{m} 1} t\right)\left(\hat{\boldsymbol{x}}(0)-\overline{\boldsymbol{x}}_{\mathrm{m} \mathrm{l}}\right)-\exp \left(\int_{0}^{t} \boldsymbol{A}_{\mathrm{l}}(\tau) \mathrm{d} \tau\right) \hat{\boldsymbol{\delta}}(0)\right\| \\
& =\left\|\exp \left(-\boldsymbol{K}_{\mathrm{m} 1} t\right)\left(\boldsymbol{x}(0)-\overline{\boldsymbol{x}}_{\mathrm{m} 1}\right)+\left(\exp \left(-\boldsymbol{K}_{\mathrm{m} 1} t\right)-\exp \left(\int_{0}^{t} \boldsymbol{A}_{1}(\tau) \mathrm{d} \tau\right) \hat{\boldsymbol{\delta}}(0)\right) \hat{\boldsymbol{\delta}}(0)\right\| \\
& \leq\left\|\boldsymbol{x}(0)-\overline{\boldsymbol{x}}_{\mathrm{m} 1}\right\|\left\|\exp \left(-\boldsymbol{K}_{\mathrm{m} \mathrm{l}} t\right)\right\|+\left\|\boldsymbol{\gamma}-\boldsymbol{K}_{\mathrm{m} 1}\right\|_{\max }\|\hat{\boldsymbol{\delta}}(0)\|\left\|\left(\exp \left(-\boldsymbol{K}_{\mathrm{m} \mathrm{t}} t\right)-\exp (-\gamma t)\right)\left(\boldsymbol{\gamma}-\boldsymbol{K}_{\mathrm{m} \mathrm{l}}\right)^{-1}\right\| \\
& =\left\|\boldsymbol{x}(0)-\overline{\boldsymbol{x}}_{\mathrm{m} \mathrm{l}}\right\|\left\|\exp \left(-\boldsymbol{K}_{\mathrm{m} 1} t\right)\right\|+\sigma\|\hat{\boldsymbol{\delta}}(0)\|\left\|\left(\exp \left(-\boldsymbol{K}_{\mathrm{m} \mathrm{l}} t\right)-\exp (-\gamma t)\right)\left(\boldsymbol{\gamma}-\boldsymbol{K}_{\mathrm{m} 1}\right)^{-1}\right\|
\end{aligned}
$$

where

$$
\sigma=\left\|\boldsymbol{\gamma}-\boldsymbol{K}_{\mathrm{m} 1}\right\|_{\max }=\max \left\{\left\|\boldsymbol{\gamma}-\boldsymbol{K}_{\mathrm{m}}\right\|\right\}
$$


And then, we let

$$
\boldsymbol{G}(t)=\exp \left(-\boldsymbol{K}_{\mathrm{m} 1 t} t\right)-\exp (-\gamma t)
$$

According to Eq. (5.73), we can obtain

$$
\frac{\mathrm{d} \boldsymbol{G}(t)}{\mathrm{d} t}=-\boldsymbol{K}_{\mathrm{m} 1} \exp \left(-\boldsymbol{K}_{\mathrm{m} 1} t\right)+\gamma \exp (-\gamma t)
$$

Then, we use the method of resolvent matrix to solve $\exp \left(-\boldsymbol{K}_{\mathrm{m}} t\right)$ and $\exp (-\boldsymbol{\beta} t)$ [12]. Thus, we have

$$
\begin{aligned}
& \exp \left(-\boldsymbol{K}_{\mathrm{m}} t\right)=\exp \left(\boldsymbol{A}_{\mathrm{m}} t\right)=\left[\begin{array}{ll}
f_{11}(t) & f_{12}(t) \\
f_{13}(t) & f_{14}(t)
\end{array}\right] \\
& \exp (-\gamma t)=\left[\begin{array}{ll}
r_{1}(t) & r_{2}(t) \\
r_{3}(t) & r_{4}(t)
\end{array}\right]=L^{-1}\left((s \boldsymbol{I}+\gamma)^{-1}\right)
\end{aligned}
$$

We let

$$
\frac{\mathrm{d} \boldsymbol{G}(t)}{\mathrm{d} t}=-\boldsymbol{K}_{\mathrm{m} 1} \exp \left(-\boldsymbol{K}_{\mathrm{m} 1} t\right)+\gamma \exp (-\gamma t)=0
$$

So, we get

$$
\boldsymbol{T}_{\mathrm{m} 1}=\left[\begin{array}{cc}
t_{11} & t_{12} \\
t_{13} & t_{14}
\end{array}\right]
$$

where $t_{11}, t_{12}, t_{13}$ and $t_{14}$ satisfy the following equations, respectively.

$$
\begin{aligned}
& \gamma_{1} r_{1}\left(t_{11}\right)+\gamma_{2} r_{3}\left(t_{21}\right)-k_{11} f_{11}\left(t_{11}\right)-k_{12} f_{13}\left(t_{11}\right)=0 \\
& \gamma_{1} r_{2}\left(t_{12}\right)+\gamma_{2} r_{4}\left(t_{12}\right)-k_{11} f_{12}\left(t_{12}\right)-k_{12} f_{14}\left(t_{12}\right)=0 \\
& \gamma_{3} r_{1}\left(t_{13}\right)+\gamma_{4} r_{3}\left(t_{13}\right)-k_{13} f_{11}\left(t_{13}\right)-k_{14} f_{13}\left(t_{13}\right)=0 \\
& \gamma_{3} r_{2}\left(t_{14}\right)+\gamma_{4} r_{4}\left(t_{14}\right)-k_{13} f_{12}\left(t_{14}\right)-k_{14} f_{14}\left(t_{14}\right)=0
\end{aligned}
$$

where $\left[\begin{array}{ll}\gamma_{1} & \gamma_{2} \\ \gamma_{3} & \gamma_{4}\end{array}\right]=\gamma$

And then, we have

$$
t_{\mathrm{m} 1}=\max \left\{\boldsymbol{T}_{\mathrm{m} 1}\right\}=\max \left\{\left[\begin{array}{ll}
t_{11} & t_{12} \\
t_{13} & t_{14}
\end{array}\right]\right\} \approx m_{11} \Delta t
$$

The proof processes of Eq. (5.63) and Eq. (5.64) are similar to the proof process of Eq. (5.62). Thus, here the proof processes are ignored.

End

In the area (1), the control strategy on checking rate is to apply (5.28) for time interval larger than $m_{12} t_{\mathrm{m} 1}$ to control the course of $|\hat{\boldsymbol{x}}(t)-\hat{\boldsymbol{x}}(t)|$, where $m_{12}$ is a suitable integer. If the difference can not remain bounded, then the estimation state probability (5.27) must be reinitialized with 
where $m_{1}=m_{11} \bullet m_{12}$.

$$
\hat{\boldsymbol{x}}\left(m_{1} \Delta t\right)=\hat{\boldsymbol{x}}\left(m_{1} \Delta t\right)
$$

In the area (2), the control strategy on checking rate is to apply (5.29) for time interval larger than $m_{22} t_{\mathrm{m} 2}$ to control the course of $|\hat{\boldsymbol{x}}(t)-\hat{x}(t)|$, where $m_{22}$ is a suitable integer. If the difference can not remain bounded, then the estimation state probability (5.27) must be reinitialized with

$$
\hat{\boldsymbol{x}}\left(m_{2} \Delta t\right)=\hat{\boldsymbol{x}}\left(m_{2} \Delta t\right)
$$

where $m_{2}=m_{21} \cdot m_{22}$.

In the area (3), the control strategy on checking rate is to apply (5.30) for time interval larger than $m_{32} t_{\mathrm{m} 3}$ to control the course of $|\hat{\boldsymbol{x}}(t)-\hat{\boldsymbol{x}}(t)|$, where $m_{32}$ is a suitable integer. If the difference can not remain bounded, then the estimation state probability (5.27) must be reinitialized with

$$
\hat{\boldsymbol{x}}\left(m_{3} \Delta t\right)=\hat{\boldsymbol{x}}\left(m_{3} \Delta t\right)
$$

where $m_{3}=m_{31} \cdot m_{32}$.

\section{Conclusion}

In this paper, the dynamic behavior of the maintenance system based on state transition is analyzed by establishing the differential equations that are used to describe the dynamic behavior of the maintenance system, and the performance indexes including the steadystate availability and the MTTF of the maintenance system are calculated and given out. Theoretical analysis shows that we can control the dynamic behavior of the maintenance system to achieve the expected steady-state performance so that we can guarantee reliable completion of the target task and modify the MTTF of the maintenance system by selecting checking rate as control variable so that we can reduce the cost of corrective maintenance. Moreover, the paper still performs devices state evaluation, and analyzes the control errors and the selection of control timing so that we can more precisely and effectively control the actual maintenance system. The results indicate that the strategy proposed in the paper that selecting checking rate as control variable to control the dynamic behavior of the maintenance system is very effective and accurate. Compared with the existing traditional PM strategy and CBM strategy, this maintenance strategy proposed in the paper has many advantages that its mathematical model is simple, its control laws on the checking rate is dynamic and real-time and it is easy to collect data as well as this strategy can be applied practically. Thus, this maintenance strategy effectively saves the inspection maintenance cost, guarantees the system steady-state availability and is able to provide the theoretical support for management problems of the large complex equipments.

\section{Acknowledgements}

This project is supported by the National Natural Foundation of China (Grant No.61263004) and Gansu Province Natural Science Foundation (Grant No.1212RJZA071).

\section{References}

[1] X. H. Yin, Y. L. Qian, Y. M. Yang, et al, "Density evolution analysis of dynamic behavior of maintenance system", China Mechanical Engineering. vol. 19, no. 8, (2008), pp. 914-918.

[2] S. V. Amari, L. McLaughlin, "Optimal design of a condition-based maintenance model", In Reliability and Maintainability, 2004 Annual Symposium-RAMS. IEEE. pp. 528-533 (2004)

[3] P. A. Scarf, "A framework for condition monitoring and condition based maintenance", Quality Technology \& Quantitative Management. vol. 4, no. 2, (2007), pp. 301-312.

[4] H. F. Zuo, J. Cai, H. W. Wang, et al, Decision Theory and Method of Maintenance, Aviation Industry Press, Beijing (2008). 
[5] A. K. S. Jardine, T. Joseph, and D. Banjevic, "Optimizing condition-based maintenance decisions for equipment subject to vibration monitoring", Journal of Quality in Maintenance Engineering. vol. 5, no. 3, (1999), pp. 192-202.

[6] R. D. Baker, and A. H. Christer, "Review of delay-time OR modeling of engineering aspects of maintenance", European Journal of Operational Research. vol. 73, no. 3, (1994), pp. 407-422.

[7] W. Wang, D. Banjevic, and M. Pecht, "A multi-component and multi-failure mode inspection model based on the delay time concept", Reliability Engineering \& System Safety. vol. 95, no. 8, (2010), pp. 912-920.

[8] C. T. Lam, and R. H. Yeh, "Optimal maintenance-policies for deteriorating systems under various maintenance strategies", IEEE Transactions on Reliability. vol. 43, no. 3, (1994), pp. 423-430.

[9] H. S. Su, "Control strategy on preventive maintenance of repairable device", Journal of Zhejiang University. vol. 44, no. 7, (2010), pp. 1308-1314.

[10] International Electrotechnical Commission, IEC 61165: Application of Markov Techniques (2006).

[11] J. H. Cao and K. Cheng, Introduction of Reliability Mathematics, Higher Education Press, Beijing (2006).

[12] D. Z. Zheng, Linear System Theory, Tsinghua University Publishing House, Beijing (2002).

[13] Q. F. Su and X. W. Li, "Judgment of matrix eigenvalue real part", Journal of Qiqihar University. vol. 16, no. 4, (2000), pp. 21-23.

[14] A. Papoulis, Probability, Random Variables, and Stochastic Processes, McGrow-Hill, New York (1991).

[15] D. H. Shi, X. J. Zeng, "Interval stability and robust stability for linear time-varying systems"Control Theory and Applications. vol. 11, no. 1, (1994), pp. 34-39.

[16] X. X. Liao, Stability Mathematical Theory and Application, Central China Normal University Press, Wuhan, (1988).

[17] S. Axler, F. W. Gehring, and P. R. Halmos, "Matrix Analysis", Springer-verlag, New York (1997).

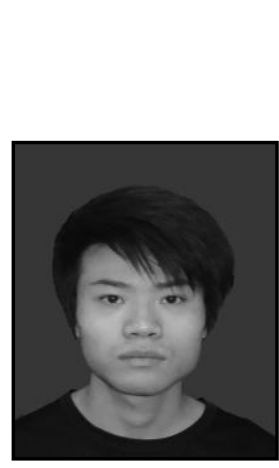

\section{Authors}

Yongqiang Kang, he is pursuing a Master's degree in Electrical Engineering, school of Automation and Electrical Engineering, Lanzhou Jiaotong University. His research interest includes Automatic Control and Reliability Engineering, System Security and Reliability, Power Systems and Its Automation, and etc.

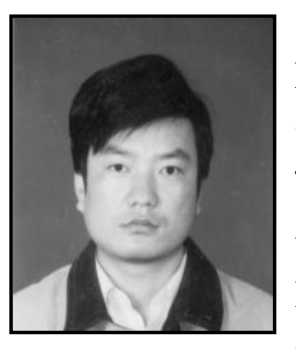

Hongsheng Su, he obtained his Master in Traffic Information Engineering and Control, Lanzhou Jiaotong University in 2001. He acquired his $\mathrm{PhD}$ in Power Systems and Its Automation, Southwest Jiaotong University. Now he is serving as a full-time professor at school of Automation and Electrical Engineering, Lanzhou Jiaotong University. His research interest includes System Security and Reliability, Intelligent Control, Power Systems and Its Automation, and etc. 